\title{
Synthesis and anti-inflammatory activity of 3-indolyl pyridine derivatives through one-pot multi component reaction
}

\author{
PRAKASAM THIRUMURUGAN, S MAHALAXMI and PARAMASIVAN T PERUMAL* \\ Organic Chemistry Division, Central Leather Research Institute, Adyar, Chennai 600020 \\ e-mail: ptperumal@gmail.com
}

MS received 24 November 2009; revised 27 May 2010; accepted 25 June 2010

\begin{abstract}
A simple protocol for the efficient preparation of 2-(1H-Indol-3-yl)-6-methoxy-4-arylpyridine-3,5-dicarbonitrile has been achieved through one-pot multi-component reaction under reflux condition. These compounds showed a good anti-inflammatory activity. Also a series of bis-Hantzsch dihydropyridine derivatives were synthesized and they exhibit analgesic activity.
\end{abstract}

Keywords. Multi component reaction; 3-cyanoacetyl indole; malononitrile; pyridine synthesis; antiinflammatory activity; analgesic activity.

\section{Introduction}

Functionalized nitrogen-heterocycles play a predominant role in medicinal chemistry and they have been intensively used as scaffolds for drug development. Multi-component reactions (MCR's) have emerged as a powerful tool for delivering the molecular diversity needed in the combinatorial approaches for the preparation of bioactive heterocyclic compounds. ${ }^{1}$ Multi-component reactions, such as the Biginelli, ${ }^{2}$ Passerini, ${ }^{3}$ Ugi, ${ }^{4}$ and Hantzsch, provide a wide variety of important heterocycles. ${ }^{5}$ For example, the Hantzsch reaction provides dihydropyridines with activity against calcium channels, multidrug resistance (MDR) proteins, 5-hydroxytryptamine $(5-\mathrm{HT})$ receptors, and anti-inflammatory targets. ${ }^{6-7}$

The pyridine nucleus is prevalent in numerous natural products and is extremely important in chemistry of biological systems. ${ }^{8}$ It plays a key role catalysing both biological and chemical systems. In many enzymes of living organisms it is the prosthetic pyridine nucleotide (NADP) that is involved in various oxidation-reduction processes. Other evidence of the potent activity of pyridine in biological systems is its presence in the important vitamins niacin and pyridoxine (vitamin B6) and also in highly toxic alkaloids such as nicotine..$^{9-11}$ The pyridine substructure is one of the most important heterocycles found in natural products, pharmaceuticals,

*For correspondence and functional materials. ${ }^{12}$ Pyridine derivatives containing multi-functional groups such as streptonigrin, streptonigrone and lavendamycin are reported as anticancer drugs, and cerivastatin is reported as the HMG-CoA enzyme inhibitor. ${ }^{8}$ Moreover, substituted pyridines are reported as leukotriene B-4 antagonists. ${ }^{13-15}$

3-Substituted indole is the one of the "privileged medicinal scaffold,' found in many biologically active compounds and natural products. ${ }^{16,17}$ Through appropriate functional group modifications, these scaffolds are capable of providing ligands for a number of functionally and structurally discrete biological receptors. 3-Substitued indole scaffolds are found in a number of biologically active compounds especially with anticancer, anti-tumour, ${ }^{18}$ hypoglycemic, anti-inflammatory, analgesic and anti-pyretic activities (figure 1). ${ }^{19-20}$

The wide-ranging biological activity associated with many pyridine and 3-substituted indole derivatives, both naturally occurring and synthetic, ensures that the synthesis of this important ring system remains a topic of current interest. Various methods for the preparation of these compounds have been reported. However, these methods suffer from tedious synthetic routes, longer reaction time, drastic reaction conditions, as well as narrow substrate scope. ${ }^{21-25}$ There have been very few reports about the synthesis of indol-3-yl derivatives including pyridine moieties. ${ }^{9,18,22-23,26}$ As part of our ongoing research on the development of novel synthetic routes for the synthesis of biologically active hetero- 
<smiles>[R2]c1c([R3])c([R])c2c(-c3ccnc(N)n3)c[nH]c2c1[R]</smiles>

Meridianins A - E<smiles>[R]c1ccc2c(-c3cnc(-c4c[nH]c5cc([R])ccc45)[nH]3)c[nH]c2c1</smiles>

Nortosentins A-C<smiles>[R]c1cccc2c(-c3ccc(-c4c[nH]c5ccccc45)s3)c[nH]c12</smiles>

2, 5 bis(3-indolyl)thiophenes<smiles></smiles>

2, 4 bis(3-indolyl)pyrimidines<smiles>O=C1NCC(c2c[nH]c3cc(Br)ccc23)N=C1c1c[nH]c2cc(Br)ccc12</smiles>

Hamacanthin B<smiles>COc1ccc2c(c1)c(CC(=O)O)c(C)n2C(=O)c1ccc(C)cc1</smiles>

Indomethacin

Figure 1. Representatives of 3-substituted indole derivatives

cyclic compounds and use of green chemical techniques in organic synthesis, ${ }^{27,28}$ we report here a simple and facile one pot procedure for the synthesis of indol-3-yl pyridine derivatives in aqueous media under reflux condition.

\section{Experimental}

\subsection{General}

All the substituted aldehydes, malononitrile, 2indanone, indole, $\mathrm{CDCl}_{3}$ and DMSO- $d_{6}$ were purchased from Aldrich Chemicals. Acetic anhydride and all other reagents were purchased from S.D. Fine Chemicals Limited and were used as received. Methanol was distilled from $\mathrm{Mg} / \mathrm{I}_{2}$ under nitrogen and stored over $3 \AA$ molecular sieves purchased from Aldrich. IR measurements were done as $\mathrm{KBr}$ pellets for solids using Perkin Elmer Spectrum RXI FT-IR. The ${ }^{1} \mathrm{H}$ and ${ }^{13} \mathrm{C}$ NMR spectra were recorded in $\mathrm{CDCl}_{3}$ and DMSO- $d_{6}$ using TMS as internal standard with JEOL $500 \mathrm{MHz}$ and Bruker $500 \mathrm{MHz}$ high resolution NMR spectrometer. Multiplicities were abbreviated as follows: singlet $(s)$, doublet $(d)$, triplet $(t)$, multiplet $(m)$, and broad $(b r)$. The mass spectra were recorded using a electrospray ionisation method with thermo Finnigan mass spectrometer. Melting points were determined in capillary tubes and are uncorrected. Analytical TLC was performed on precoated plastic sheets of silica gel G/UV-254 of $0.2 \mathrm{~mm}$ thickness (Macherey-Nagel, Germany). Elemental analysis data were recorded using Thermo Finnigan FLASH EA 1112 CHN instrument.

2.2 General procedure for the synthesis of indol-3$y$ l pyridine and 2,2'-bipyridine derivatives (4a-p)

A mixture of 3-cyanoacetyl indole $(1.0 \mathrm{mmol})$, aldehyde $(1.0 \mathrm{mmol})$, malononitrile $(1.0 \mathrm{mmol})$ and freshly prepared sodium methoxide $(1.0 \mathrm{mmol})$ (or) sodium hydroxide $(1.2 \mathrm{mmol})$ in $30 \mathrm{~mL}$ of methanol: water $(2: 1)$ was refluxed. After the completion of the reaction (as monitored by TLC), it was poured into water, filtered and then dried. The crude product was further purified by recrystallization with ethanol and the appropriate isolated yield shown in table 1 .

2.2a 2-(1H-Indol-3-yl)-6-methoxy-4-phenylpyridine-3,5-dicarbonitrile (table 1, entry 1) 4a: Light yellow solid; m.p. $244-246^{\circ} \mathrm{C} ; \quad R_{f} \quad 0.23 \quad(20 \%$ 
Table 1. Synthesis of indol-3-yl pyridine derivatives.

\begin{tabular}{|c|c|c|c|c|c|}
\hline Entry & Aldehyde (1) & Product $(4)^{\mathrm{a}}$ & & Time (h) & Yield $(\%)^{\mathrm{b}}$ \\
\hline 1 & & & & $1 \cdot 5$ & 89 \\
\hline 2 & & & & $1 \cdot 0$ & 91 \\
\hline 3 & & & $4 c$ & 1.5 & 86 \\
\hline 4 & & & & $1 \cdot 5$ & 91 \\
\hline 5 & & & & $2 \cdot 5$ & 79 \\
\hline 6 & & & & $2 \cdot 0$ & 81 \\
\hline 7 & & & & $2 \cdot 0$ & 81 \\
\hline 8 & & & & $2 \cdot 0$ & 79 \\
\hline 9 & & & & $2 \cdot 0$ & 79 \\
\hline 10 & & & Ai & $1 \cdot 0$ & 81 \\
\hline 11 & & & & $3 \cdot 0$ & 72 \\
\hline 12 & & & & $2 \cdot 5$ & 68 \\
\hline
\end{tabular}


Table 1. (Contd...)

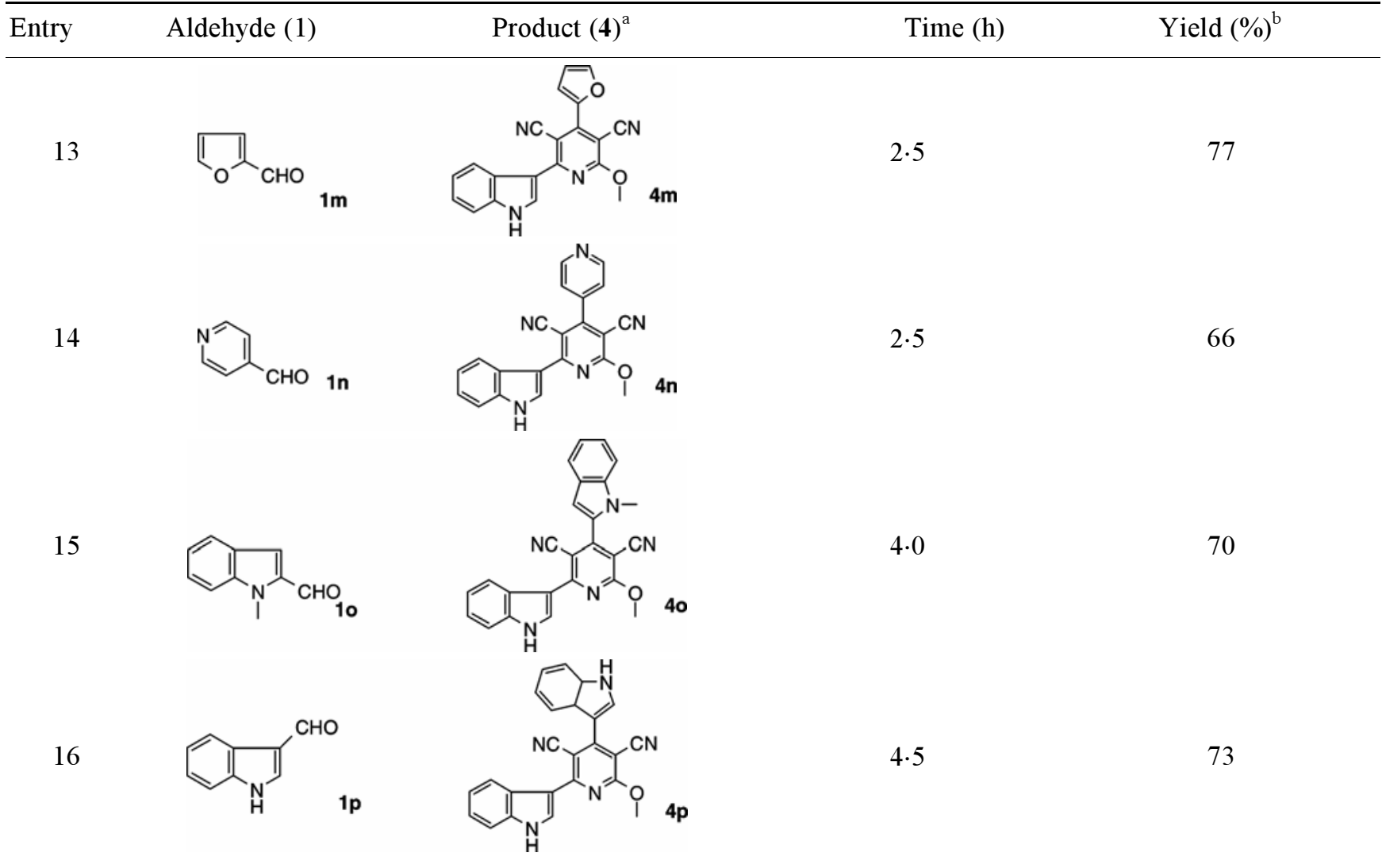

${ }^{a}$ All the products were characterized by NMR, IR and mass spectroscopy. ${ }^{b}$ Isolated yield

EtOAc/petroleum ether); IR ( $\mathrm{KBr}): 1153,1229$, 1427, 1516, 1629, 2230, $3287 \mathrm{~cm}^{-1}$; ${ }^{1} \mathrm{H}$ NMR $\left(500 \mathrm{MHz}, \mathrm{DMSO}-d_{6}\right): \delta 4 \cdot 21\left(s, 3 \mathrm{H},-\mathrm{OCH}_{3}\right), 7 \cdot 22-$ $7.24(m, 2 \mathrm{H},-\operatorname{Ar}-H), 7.52-7.60(m, 6 \mathrm{H},-\operatorname{Ar}-H)$, 8.43-8.45 (m, 1H, $-\mathrm{Ar}-H), 8.57(s, 1 \mathrm{H},-\mathrm{Ar}-H)$, $12.17(b r s, 1 \mathrm{H},-\mathrm{NH}) ;{ }^{13} \mathrm{C}$ NMR $(125 \mathrm{MHz}$, DMSO$\left.d_{6}\right): \delta 56 \cdot 2,91 \cdot 7,97 \cdot 0,112 \cdot 7,113 \cdot 0,114 \cdot 6,118 \cdot 3$, $122 \cdot 2,122 \cdot 6,123 \cdot 5,126 \cdot 4,128 \cdot 8,129 \cdot 1,129 \cdot 2$, $130 \cdot 9,132 \cdot 1,134 \cdot 5,137 \cdot 2,159 \cdot 3,161 \cdot 0,164 \cdot 8$; MS (ESI LCQ-MS): $m / z 351.20[\mathrm{M}+\mathrm{H}]^{+}$. Anal. Calcd. for $\mathrm{C}_{22} \mathrm{H}_{14} \mathrm{~N}_{4} \mathrm{O}$ : C $75.42 \mathrm{H} 4.03 \mathrm{~N} \mathrm{15.99}$. Found: $\mathrm{C}$ $75.31 \mathrm{H} 4.02 \mathrm{~N} 16.04$.

2.2b 2-(1H-Indol-3-yl)-6-methoxy-4-(4-tolyl phenyl) pyridine-3,5-dicarbonitrile (table 2, entry 2) $\mathbf{4 b}$ : Light yellow solid; m.p. $228-230^{\circ} \mathrm{C} ; R_{f} 0.30(20 \%$ EtOAc/Petroleum ether); IR (KBr): 1145, 1224, 1306, 1422, 1516, 1620, 2221, $3325 \mathrm{~cm}^{-1}{ }^{1} \mathrm{H}$ NMR $\left(500 \mathrm{MHz}, \mathrm{DMSO}-d_{6}\right): \delta 2.41\left(s, 3 \mathrm{H},-\mathrm{Ar}-\mathrm{CH}_{3}\right) 4.21$ $\left(s, 3 \mathrm{H},-\mathrm{OCH}_{3}\right), 7 \cdot 19-7.24(m, 2 \mathrm{H},-\mathrm{Ar}-\mathrm{H}), 7 \cdot 36-$ $7.52(m, 5 \mathrm{H},-\operatorname{Ar}-H), 8.42(d, 1 \mathrm{H}, J=8.4 \mathrm{~Hz},-\mathrm{Ar}-$ $H), 8.55(\mathrm{~s}, 1 \mathrm{H},-\mathrm{Ar}-\mathrm{H}), 12.19(\mathrm{brs}, 1 \mathrm{H},-\mathrm{NH}){ }^{13} \mathrm{C}$ NMR (125 MHz, DMSO- $\left.d_{6}\right): \delta 21 \cdot 0,55 \cdot 5,91 \cdot 3$,
$96 \cdot 7, \quad 112 \cdot 2,112 \cdot 3,114 \cdot 0,117 \cdot 7,121 \cdot 6,122 \cdot 0$, $122.9,125.9,128 \cdot 2,128 \cdot 5,129 \cdot 2,131 \cdot 0,131 \cdot 1$, $136 \cdot 4,140 \cdot 2,159 \cdot 0,160 \cdot 5,164 \cdot 3$. MS (ESI LCQMS): $m / z \quad 365 \cdot 20 \quad[\mathrm{M}+\mathrm{H}]^{+}$. Anal. Calcd. for $\mathrm{C}_{23} \mathrm{H}_{16} \mathrm{~N}_{4} \mathrm{O}$ : C $75.81 \mathrm{H} 4.43 \mathrm{~N}$ 15.38. Found: $\mathrm{C}$ $75.90 \mathrm{H} 4.42 \mathrm{~N} 15.33$.

2.2c 2-(1H-Indol-3-yl)-6-methoxy-4-(3-methylphenyl)pyridine-3,5-dicarbonitrile (table 1, entry 3) 4c: Pale yellow solid; m.p. $286-288^{\circ} \mathrm{C} ; R_{f} 0.23$ (20\% EtOAc/petroleum ether); IR (KBr): 1163 , $1237,1358,1434,1553,1617,2366,3262 \mathrm{~cm}^{-1}$ ${ }^{1} \mathrm{H}$ NMR $\left(500 \mathrm{MHz}\right.$, DMSO- $\left.d_{6}\right): \delta 2.37 \quad(s, 3 \mathrm{H}$, $\left.-\mathrm{Ar}-\mathrm{CH}_{3}\right) 4.18\left(\mathrm{~s}, 3 \mathrm{H},-\mathrm{OCH}_{3}\right), 7 \cdot 20-7.26(m, 2 \mathrm{H}$, $-\mathrm{Ar}-H), 7.34-7.36(m, 3 \mathrm{H},-\mathrm{Ar}-H), 7.43(t, 1 \mathrm{H}$, $J=7.6 \mathrm{~Hz},-\operatorname{Ar}-H), 7.52(d, 1 \mathrm{H}, J=7.6 \mathrm{~Hz},-\operatorname{Ar}-H)$, $8.42(d, 1 \mathrm{H}, J=8.4 \mathrm{~Hz},-\operatorname{Ar}-H), 8.55(s, 1 \mathrm{H},-\mathrm{Ar}-$ $H), 12.14(b r s, 1 \mathrm{H},-\mathrm{NH}) ;{ }^{13} \mathrm{C}$ NMR $(125 \mathrm{MHz}$, DMSO- $\left.d_{6}\right): \delta 20 \cdot 9,55 \cdot 7,91 \cdot 3,96 \cdot 6,112 \cdot 2,112 \cdot 4$, $114 \cdot 1,117 \cdot 7,121 \cdot 7,122 \cdot 1,123 \cdot 0,125 \cdot 7,125 \cdot 8$, $128 \cdot 6,128 \cdot 9,131 \cdot 0,131 \cdot 3,133 \cdot 9,136 \cdot 5,138 \cdot 0$, $158.9,160 \cdot 7,164 \cdot 3$. MS (ESI LCQ-MS): $\mathrm{m} / \mathrm{z}$ $365.20[\mathrm{M}+\mathrm{H}]^{+}$. Anal. Calcd. for $\mathrm{C}_{23} \mathrm{H}_{16} \mathrm{~N}_{4} \mathrm{O}$ : 


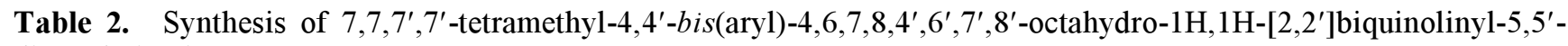
dione derivatives

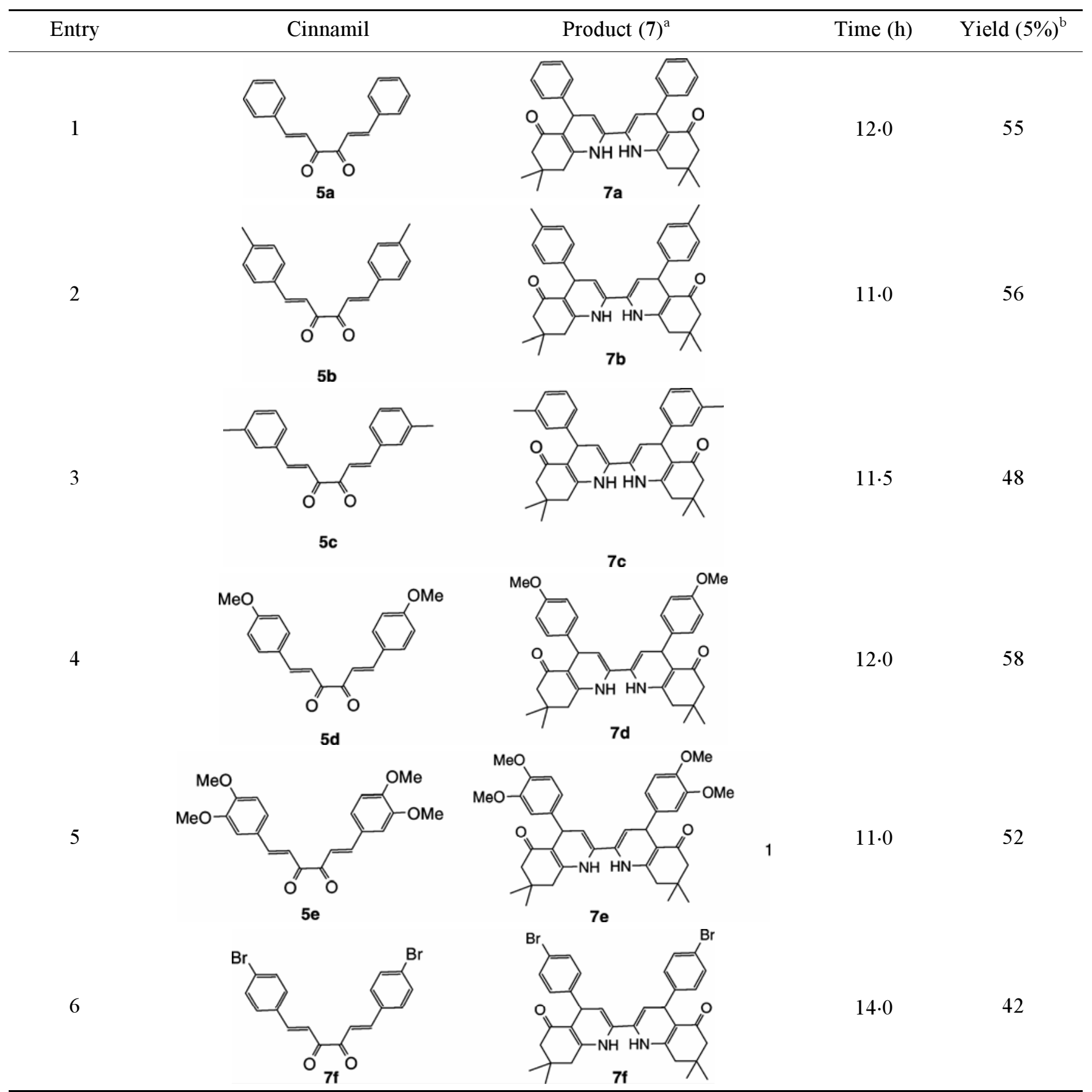

${ }^{\mathrm{a}}$ The products were characterized by IR, NMR, mass and elemental analysis. ${ }^{\mathrm{b}}$ Isolated yield.

C $75.81 \mathrm{H} 4.43 \mathrm{~N} 15 \cdot 38$. Found: C $75.72 \mathrm{H} 4.44 \mathrm{~N}$ $15 \cdot 43$.

2.2d 2-(1H-Indol-3-yl)-6-methoxy-4(4methoxyphenyl) pyridine -3,5-dicarbonitrile (table 1, entry 4) 4d: Yellow solid; m.p. $268-270{ }^{\circ} \mathrm{C} ; R_{f} 0.23(20 \%$ EtOAc/petroleum ether); IR (KBr): 1163, 1237,
$1358,1434,1553,1617,2366,3262 \mathrm{~cm}^{-1}{ }^{1} \mathrm{H}$ NMR $\left(500 \mathrm{MHz}, \mathrm{DMSO}-d_{6}\right): \delta 3.80\left(s, 3 \mathrm{H},-\mathrm{OCH}_{3}\right), 4 \cdot 20$ $\left(s, 3 \mathrm{H},-\mathrm{OCH}_{3}\right), 7.06(d, 2 \mathrm{H}, J=8.4 \mathrm{~Hz},-\mathrm{Ar}-H)$, $7 \cdot 12(d, 1 \mathrm{H}, J=8 \cdot 2 \mathrm{~Hz},-\mathrm{Ar}-H), 7 \cdot 19-7.22(m, 1 \mathrm{H}$, $-\operatorname{Ar}-H), 7.43(d, 2 \mathrm{H}, J=8.4 \mathrm{~Hz},-\operatorname{Ar}-H), 7.56(d$, $1 \mathrm{H}, J=8.4 \mathrm{~Hz},-\mathrm{Ar}-H), 8.41-8.43(m, 1 \mathrm{H},-\mathrm{Ar}-H)$, $8.58(s, 1 \mathrm{H},-\mathrm{Ar}-H), 12.31($ brs $, 1 \mathrm{H},-\mathrm{N} H) ;{ }^{13} \mathrm{C}$ 
NMR $\left(125 \mathrm{MHz}\right.$, DMSO- $\left.d_{6}\right): \delta 55 \cdot 2,55 \cdot 7,91 \cdot 1$, $96 \cdot 4,112 \cdot 2,112 \cdot 7,114 \cdot 1,117 \cdot 7,121 \cdot 7,122 \cdot 0$, $122.9,126 \cdot 0,128.9,130 \cdot 7,131.9,132 \cdot 8,135 \cdot 4$, 137.0, 158.8, 159.4, 164.2. MS (ESI LCQ-MS): $\mathrm{m} / \mathrm{z}$ $381.27[\mathrm{M}+\mathrm{H}]^{+}$. Anal. Calcd. for $\mathrm{C}_{23} \mathrm{H}_{16} \mathrm{~N}_{4} \mathrm{O}_{2}$ : C

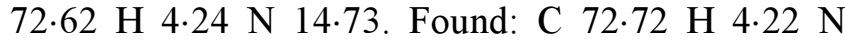
14.69 .

\section{2e 4-(2-Chlorophenyl)-2-(1H-indol-3-yl)-6-}

methoxypyridine-3,5-dicarbonitrile (table 1 , entry 5) 4e: Pale yellow solid; m.p. $214-216^{\circ} \mathrm{C} ; R_{f} 0.31$ (20\% EtOAc/petroleum ether); IR (KBr): 1090, $1145,1221,1420,1519,2219,3325 \mathrm{~cm}^{-1}{ }^{1} \mathrm{H}$ NMR $\left(500 \mathrm{MHz}, \mathrm{DMSO}-d_{6}\right): \delta 4 \cdot 25\left(s, 3 \mathrm{H},-\mathrm{OCH}_{3}\right), 7 \cdot 23-$ $7.26(m, 2 \mathrm{H},-\operatorname{Ar}-H), 7.53-7.63(m, 4 \mathrm{H},-\operatorname{Ar}-H)$, $7.71(d, 1 \mathrm{H}, J=8.4 \mathrm{~Hz},-\operatorname{Ar}-H), 8.47-8.49(m, 1 \mathrm{H}$, $-\mathrm{Ar}-H), 8.58(\mathrm{~s}, 1 \mathrm{H},-\mathrm{Ar}-H), 12.41(\mathrm{brs}, 1 \mathrm{H},-\mathrm{N} H)$; ${ }^{13} \mathrm{C}$ NMR (125 MHz, DMSO- $\left.d_{6}\right): \delta 55 \cdot 9,91 \cdot 9,97 \cdot 0$, $112 \cdot 1,112 \cdot 6,113 \cdot 4,116 \cdot 8,121 \cdot 9,122 \cdot 1,123 \cdot 2$, $125 \cdot 7,127.9,129.9,130.4,131 \cdot 0,131.5,132 \cdot 1$, $133 \cdot 0,136 \cdot 6,158 \cdot 3,158 \cdot 8,164 \cdot 2$. MS (ESI LCQMS): $m / z \quad 385 \cdot 17 \quad[\mathrm{M}+\mathrm{H}]^{+}$. Anal. Calcd. for $\mathrm{C}_{22} \mathrm{H}_{13} \mathrm{ClN}_{4} \mathrm{O}$ : C $68.67 \mathrm{H} \mathrm{3.41} \mathrm{N} \mathrm{9.21.} \mathrm{Found:} \mathrm{C}$ $68.75 \mathrm{H} 3.40 \mathrm{~N} 9 \cdot 17$.

\section{$2.2 \mathrm{f}$ 4-(4-Chlorophenyl)-2-(1H-indol-3-yl)-6-}

methoxypyridine-3,5-dicarbonitrile (table 1, entry 6) 4f: Pale yellow solid; m.p. $260-262^{\circ} \mathrm{C} ; R_{f} 0.31$ (20\% EtOAc/petroleum ether); IR (KBr): 1093 , $1222,1366,1423,1490,1517,1573,2222$, $3329 \mathrm{~cm}^{-1}{ }^{1} \mathrm{H}$ NMR $\left(500 \mathrm{MHz}, \mathrm{DMSO}-d_{6}\right): \delta 4.21$ $\left(s, 3 \mathrm{H},-\mathrm{OCH}_{3}\right), 7.23-7.25(m, 2 \mathrm{H},-\mathrm{Ar}-H), 7.53(d$, $1 \mathrm{H}, J=8.4 \mathrm{~Hz},-\operatorname{Ar}-H), 7.62(q, 4 \mathrm{H}, J=8.4 \mathrm{~Hz},-$ $\operatorname{Ar}-H) 8.43(d, 1 \mathrm{H}, J=8.4 \mathrm{~Hz},-\operatorname{Ar}-H), 8.56(s, 1 \mathrm{H},-$ Ar- $H), 12 \cdot 15(b r s, 1 \mathrm{H},-\mathrm{N} H) ;{ }^{13} \mathrm{C}$ NMR $(125 \mathrm{MHz}$, DMSO- $\left.d_{6}\right): \delta 55 \cdot 7,91 \cdot 3,96 \cdot 6,112 \cdot 1,112 \cdot 4,114 \cdot 0$, $117 \cdot 6,121 \cdot 8,122 \cdot 0,123 \cdot 0,125 \cdot 6,128 \cdot 8,130 \cdot 2$, $130 \cdot 6,131 \cdot 3,132 \cdot 7,135 \cdot 4,136 \cdot 5,158 \cdot 8,159 \cdot 3$, 164.2; MS (ESI LCQ-MS): $m / z \quad 385.20[\mathrm{M}+\mathrm{H}]^{+}$. Anal. Calcd. for $\mathrm{C}_{22} \mathrm{H}_{13} \mathrm{ClN}_{4} \mathrm{O}: \mathrm{C} 68.67 \mathrm{H} 3.41 \mathrm{~N}$ 9.21. Found: C 68.59 H 3.42 N 9.25.

\section{$2.2 \mathrm{~g}$ 4-(2,4-Chlorophenyl)-2-(1H-indol-3-yl)-6-} methoxy pyridine-3,5-dicarbonitrile (table 1, entry 7) 4g: Pale yellow solid; m.p. $272-274^{\circ} \mathrm{C} ; R_{f} 0.31$ (20\% EtOAc/petroleum ether); IR (KBr): 1145 , $1225,1310,1422,1522,2232,3313 \mathrm{~cm}^{-1}{ }^{1} \mathrm{H} \mathrm{NMR}$ $\left(500 \mathrm{MHz}, \mathrm{DMSO}-d_{6}\right): \delta 4.25\left(s, 3 \mathrm{H},-\mathrm{OCH}_{3}\right), 7 \cdot 25-$ $7.26(m, 2 \mathrm{H},-\operatorname{Ar}-H), 7.54-7.56(m, 1 \mathrm{H},-\operatorname{Ar}-H)$, 7.66-7.71 ( $m, 2 \mathrm{H},-\operatorname{Ar}-H), 7.96(d, 1 \mathrm{H}, J=1.55 \mathrm{~Hz}$, -Ar- $H$ ) 8.46-8.48 ( $m, 1 \mathrm{H},-\mathrm{Ar}-H), 8.58(s, 1 \mathrm{H},-\mathrm{Ar}-$
$H), 12 \cdot 22(b r s, 1 \mathrm{H},-\mathrm{N} H) ;{ }^{13} \mathrm{C}$ NMR $(125 \mathrm{MHz}$, DMSO- $\left.d_{6}\right): \delta 55.9,91 \cdot 8,96 \cdot 7,112 \cdot 0,112.5,113 \cdot 3$, $116 \cdot 8,121 \cdot 9,122 \cdot 1,123 \cdot 2,125 \cdot 7,128 \cdot 2,129 \cdot 5$, $131.5,131 \cdot 8,131.9,132 \cdot 3,136 \cdot 1,136 \cdot 5,157 \cdot 2$, $158 \cdot 8, \quad 164 \cdot 1$; MS (ESI LCQ-MS): $\mathrm{m} / z$ 419.13 $[\mathrm{M}+\mathrm{H}]^{+}$. Anal. Calcd. for $\mathrm{C}_{22} \mathrm{H}_{12} \mathrm{Cl}_{2} \mathrm{~N}_{4} \mathrm{O}: \mathrm{C} 63.02$ H 2.88 N 16.91. Found: C 62.91 H 2.88 N 16.9.

\section{2h 4-(4-Bromophenyl)-2-(1H-indol-3-yl)-6-}

methoxypyridine-3,5-dicarbonitrile (table 1 , entry 8) 4h: Pale yellow solid; m.p. $238-240^{\circ} \mathrm{C} ; R_{f} 0 \cdot 31$ (20\% EtOAc/petroleum ether); IR (KBr): 1145 , $1235,1288,1435,1522,1636,2253,3222 \mathrm{~cm}^{-1}$ ${ }^{1} \mathrm{H}$ NMR $\left(500 \mathrm{MHz}\right.$, DMSO- $\left.d_{6}\right): \delta 4.22(s, 3 \mathrm{H},-$ $\left.\mathrm{OCH}_{3}\right), 7.22-7.27(m, 2 \mathrm{H},-\mathrm{Ar}-\mathrm{H}), 7.53-7.58(\mathrm{~m}$, $3 \mathrm{H},-\operatorname{Ar}-H), 7.80(d, 2 \mathrm{H}, J=8.4 \mathrm{~Hz},-\operatorname{Ar}-H) 8.43(d$, $1 \mathrm{H}, J=8.4 \mathrm{~Hz},-\operatorname{Ar}-H), 8.56(s, 1 \mathrm{H},-\operatorname{Ar}-H), 12.14$ $(b r s, 1 \mathrm{H},-\mathrm{N} H) ;{ }^{13} \mathrm{C}$ NMR $\left(125 \mathrm{MHz}\right.$, DMSO- $\left.d_{6}\right): \delta$ $55 \cdot 7,91 \cdot 3,96 \cdot 6,112 \cdot 2,112 \cdot 7,114 \cdot 2,115 \cdot 8,117 \cdot 8$, $121 \cdot 7,122 \cdot 0,123 \cdot 0,126 \cdot 0,130 \cdot 4,131 \cdot 3,131 \cdot 4$, $132 \cdot 0,137 \cdot 0,158 \cdot 8,159 \cdot 6,162 \cdot 2,164 \cdot 2,164 \cdot 3$; MS (ESI LCQ-MS): $m / z 431.13[\mathrm{M}+\mathrm{H}]+$. Anal. Calcd for $\mathrm{C}_{22} \mathrm{H}_{13} \mathrm{BrN}_{4} \mathrm{O}$ : C $61.55 \mathrm{H} 3.05 \mathrm{~N}$ 13.05. Found: $\mathrm{C}$ $61.44 \mathrm{H} 3.06 \mathrm{~N} 13.09$.

\section{$2.2 \mathrm{i}$ 4-(4-Fluorophenyl)-2-(1H-indol-3-yl)-6-me-} thoxypyridine-3,5-dicarbonitrile (table 1, entry 9) $4 \mathbf{i}$ : Pale yellow solid; m.p. $256-258^{\circ} \mathrm{C} ; R_{f} 0.30(20 \%$ EtOAc/petroleum ether); IR (KBr): 1152, 1227, 1370, 1421, 1511, 1603, 2224, $3317 \mathrm{~cm}^{-1}{ }^{1} \mathrm{H}$ NMR $\left(500 \mathrm{MHz}, \mathrm{DMSO}-d_{6}\right): \delta 4 \cdot 21\left(s, 3 \mathrm{H},-\mathrm{OCH}_{3}\right), 7 \cdot 21-$ $7.26(m, 2 \mathrm{H},-\mathrm{Ar}-H), 7.42(t, 2 \mathrm{H}, J=9.15 \mathrm{~Hz},-\mathrm{Ar}-$ $H), 7.52(d, 1 \mathrm{H}, J=6.8 \mathrm{~Hz},-\operatorname{Ar}-H) 7.67-7.69(\mathrm{~m}$, $2 \mathrm{H},-\operatorname{Ar}-H), 8.43(d, 1 \mathrm{H}, J=8.4 \mathrm{~Hz},-\operatorname{Ar}-H), 8.56$ $(s, 1 \mathrm{H},-\mathrm{Ar}-H), 12.08($ brs $, 1 \mathrm{H},-\mathrm{N} H) ;{ }^{13} \mathrm{C}$ NMR $\left(125 \mathrm{MHz}, \mathrm{DMSO}-d_{6}\right): \delta 55 \cdot 7,91 \cdot 5,96 \cdot 8,112 \cdot 1$, $114 \cdot 1,115 \cdot 7,116 \cdot 0,117 \cdot 7,121 \cdot 8,122 \cdot 1,123 \cdot 1$, $125 \cdot 8,130 \cdot 3,131 \cdot 3,131 \cdot 4,136 \cdot 5,158 \cdot 8,159 \cdot 6$, 161.6, 164.9; MS (ESI LCQ-MS): $\mathrm{m} / \mathrm{z} \quad 369.27$ $[\mathrm{M}+\mathrm{H}]^{+}$. Anal. Calcd. for $\mathrm{C}_{22} \mathrm{H}_{13} \mathrm{FN}_{4} \mathrm{O}_{7}$ : C $71.73 \mathrm{H}$ 3.56 N 15.21. Found: C 71.62 H 3.57 N 15.26.

\section{$2.2 \mathrm{j} \quad$ 2-(1H-Indol-3-yl)-6-methoxy-4-(1-naphthyl)} pyridine-3,5-dicarbonitrile (table 1 , entry 10) $\mathbf{4 j}$ : Yellow solid; m.p. $240.242^{\circ} \mathrm{C} ; R_{f} 0.21 \quad(20 \%$ EtOAc/petroleum ether); IR ( $\mathrm{KBr})$ : 1148, 1228, $1422, \quad 1514, \quad 1633, \quad 2235, \quad 3244 \mathrm{~cm}^{-1}{ }^{1} \mathrm{H}$ NMR $\left(500 \mathrm{MHz}, \mathrm{DMSO}-d_{6}\right): \delta 4 \cdot 27\left(s, 3 \mathrm{H},-\mathrm{OCH}_{3}\right), 7 \cdot 20-$ $7.28(m, 2 \mathrm{H},-\operatorname{Ar}-H), 7.47-7.70(m, 6 \mathrm{H},-\operatorname{Ar}-H)$, 8.06-8.15 ( $m, 2 \mathrm{H},-\mathrm{Ar}-H), 8.53-8.56(m, 2 \mathrm{H},-\mathrm{Ar}-$ $H), 12 \cdot 15(b r s, 1 \mathrm{H},-\mathrm{NH}) ;{ }^{13} \mathrm{C}$ NMR $(125 \mathrm{MHz}$, 
DMSO- $\left.d_{6}\right): \delta 55 \cdot 8,92 \cdot 9,97 \cdot 9,112 \cdot 2,112 \cdot 5,117 \cdot 4$, $121 \cdot 0,121 \cdot 85,122 \cdot 2,123 \cdot 2,124 \cdot 5,125 \cdot 4,126 \cdot 7$, $127 \cdot 0,127 \cdot 5,128.6,129.8,130 \cdot 4,131.4,131 \cdot 7$, $133 \cdot 0,136 \cdot 6,158 \cdot 9,159 \cdot 9,164 \cdot 4$; MS (ESI LCQMS): $m / z \quad 351.20 \quad[\mathrm{M}+\mathrm{H}]^{+}$. Anal. Calcd. for $\mathrm{C}_{26} \mathrm{H}_{16} \mathrm{~N}_{4} \mathrm{O}$ : C $75.42 \mathrm{H} 4.03 \mathrm{~N}$ 15.99. Found: $\mathrm{C}$ $75 \cdot 31 \mathrm{H} 4 \cdot 02 \mathrm{~N} 16 \cdot 04$.

\section{$2.2 \mathrm{k} \quad$ 2-(1H-Indol-3-yl)-6-methoxy-4-(3-nitrophenyl)} pyridine-3,5-dicarbonitrile (table 1, entry 11) 4k: Yellow solid; m.p. $\quad 242-244^{\circ} \mathrm{C} ; \quad R_{f} \quad 0.08 \quad(20 \%$ EtOAc/petroleum ether); IR (KBr): 1098, 1236, 1349, 1433, 1531, 1620, 2216, $3386 \mathrm{~cm}^{-1}{ }^{1} \mathrm{H}$ NMR $\left(500 \mathrm{MHz}, \mathrm{DMSO}-d_{6}\right): \delta 4 \cdot 28\left(s, 3 \mathrm{H},-\mathrm{OCH}_{3}\right), 7 \cdot 28-$ $7.31(m, 2 \mathrm{H},-\operatorname{Ar}-H), 7.57-7.59(m, 1 \mathrm{H},-\operatorname{Ar}-H)$, $7.94(t, 1 \mathrm{H}, J=7.6 \mathrm{~Hz},-\operatorname{Ar}-H), 8.14(d, 1 \mathrm{H}$, $J=7.6 \mathrm{~Hz},-\operatorname{Ar}-H), 8.48-8.50(m, 2 \mathrm{H},-\mathrm{Ar}-H), 8.62$ $(s, 2 \mathrm{H},-\mathrm{Ar}-H), 12.21(b r s, 1 \mathrm{H},-\mathrm{N} H) ;{ }^{13} \mathrm{C}$ NMR $\left(125 \mathrm{MHz}, \mathrm{DMSO}-d_{6}\right): \delta 55.9,91.5,96 \cdot 6,112 \cdot 1$, $112 \cdot 6, \quad 114 \cdot 0,117 \cdot 6,121 \cdot 9,122 \cdot 1,123 \cdot 2,123 \cdot 9$, $125 \cdot 3,125 \cdot 8,130 \cdot 7131 \cdot 6,135 \cdot 4,135 \cdot 5,136 \cdot 6$, 147.7, 158.1, 158.9, 164.2; MS (ESI LCQ-MS): $\mathrm{m} / \mathrm{z}$ $395.13[\mathrm{M}+\mathrm{H}]^{+}$. Anal. Calcd. for $\mathrm{C}_{22} \mathrm{H}_{13} \mathrm{~N}_{5} \mathrm{O}_{3}$ : C 66.83 H 3.31 N 17.71. Found: C 66.74 H $3.32 \mathrm{~N}$ $17 \cdot 75$.

\section{$2.21 \quad 2-(1 H-I n d o l-3-y l)-6-m e t h o x y-4-(2-t h i e n y l)$} pyridine-3,5-dicarbonitrile (table 1, entry 12) 4l: Yellow solid; m.p. $226-228^{\circ} \mathrm{C} ; R_{f} \quad 0.76 \quad(40 \%$ AcOEt/Petroleum ether); IR (KBr): 1134, 1226, $1475,1514,2222,3268 \mathrm{~cm}^{-1}{ }^{1} \mathrm{H}$ NMR $(500 \mathrm{MHz}$, DMSO- $\left.d_{6}\right): \delta 4 \cdot 17\left(s, 3 \mathrm{H},-\mathrm{OCH}_{3}\right), 7 \cdot 19-7 \cdot 24(\mathrm{~m}$, $1 \mathrm{H},-\operatorname{Ar}-H), 7.28(t, 1 \mathrm{H}, J=4.6 \mathrm{~Hz},-\operatorname{Ar}-H), 7.50$ $(d, 1 \mathrm{H}, J=7.6 \mathrm{~Hz},-\operatorname{Ar}-H), 7.58(d, 1 \mathrm{H}, J=3.1 \mathrm{~Hz}$, $-\operatorname{Ar}-H), 7.95(d, 1 \mathrm{H}, J=4.6 \mathrm{~Hz},-\operatorname{Ar}-H), 8.38(d$, $1 \mathrm{H}, \mathrm{J}=6.9 \mathrm{~Hz},-\operatorname{Ar}-H), 8.56(s, 1 \mathrm{H},-\operatorname{Ar}-H), 11.67$ $(b r s, 1 \mathrm{H},-\mathrm{N} H) ;{ }^{13} \mathrm{C}$ NMR $(125 \mathrm{MHz}$, DMSO-d 6 ): $\delta$ $56 \cdot 3,91 \cdot 7,97 \cdot 1,112 \cdot 6,113 \cdot 2,114 \cdot 8,118 \cdot 5,122 \cdot 6$, $123 \cdot 6,126 \cdot 5,128 \cdot 5,131 \cdot 6,132 \cdot 2,132 \cdot 6,133 \cdot 2$, 137.4, 153.6, 159.7, 165.1; MS (EI): $m / z$ 357.20 $\left[\mathrm{M}^{+}+\mathrm{H}^{+}\right]$; Anal. Calcd. for $\mathrm{C}_{20} \mathrm{H}_{12} \mathrm{~N}_{4} \mathrm{OS}$. C $67.40 \mathrm{H}$ 3.39 N 15.72. Found: C 67.31 H 3.38 N 15.74.

\section{$2.2 \mathrm{~m} \quad 4-(2-F u r y l)-2-(1 H-i n d o l-3-y l)-6-m e t h o x y-$} pyridine-3,5-dicarbonitrile (table 1, entry 13) $\mathbf{4 m :}$ Yellow solid; m.p. $234-236^{\circ} \mathrm{C} ; R_{f} \quad 0.72 \quad(40 \%$ AcOEt/Petroleum ether); IR (KBr): 1150, 1229, 1424, 2221, $3301 \mathrm{~cm}^{-1}{ }^{1} \mathrm{H}$ NMR $(500 \mathrm{MHz}$, DMSO$\left.d_{6}\right): \delta 4 \cdot 15\left(s, 3 \mathrm{H},-\mathrm{OCH}_{3}\right), 6 \cdot 84-6.86(m, 1 \mathrm{H},-\mathrm{Ar}-$ $H), 7 \cdot 17-7 \cdot 22(m, 2 \mathrm{H},-\mathrm{Ar}-H), 7 \cdot 42(d, 1 \mathrm{H}, J=3 \cdot 1$ $\mathrm{Hz},-\operatorname{Ar}-H), 7.49(d, 1 \mathrm{H}, J=7.6 \mathrm{~Hz},-\operatorname{Ar}-H), 8.11$ $(s, 1 \mathrm{H},-\mathrm{Ar}-H), 8.11(d, 1 \mathrm{H}, J=7.6 \mathrm{~Hz},-\mathrm{Ar}-H)$, $8.55(s, 1 \mathrm{H},-\mathrm{Ar}-H), 12.39(\mathrm{brs}, 1 \mathrm{H},-\mathrm{N} H) ;{ }^{13} \mathrm{C}$ NMR $\left(125 \mathrm{MHz}\right.$, DMSO- $\left.d_{6}\right): \delta 56.3,87.8,93.4$, $112 \cdot 6,113 \cdot 1,113 \cdot 4,115 \cdot 0,117 \cdot 4,118 \cdot 9,122 \cdot 2$, $122 \cdot 6,123 \cdot 5,126 \cdot 5,132 \cdot 7,137 \cdot 3,145 \cdot 6,146 \cdot 7$, 160.3, 162.8, 165.6; MS (EI): $m / z 341 \cdot 33\left[\mathrm{M}^{+}+\mathrm{H}^{+}\right]$; Anal. Calcd. for $\mathrm{C}_{20} \mathrm{H}_{12} \mathrm{~N}_{4} \mathrm{O}_{2}$ : C $67.40 \mathrm{H} 3.39 \mathrm{~N}$ 15.72. Found: C 67.31 H 3.38 N 15.74.

\section{2n 2-(1H-Indol-3-yl)-6-methoxy-4,4'-bipyridine-} 3,5-dicarbonitrile (table 1, entry 14) 4n: Yellow solid; m.p. $172-175^{\circ} \mathrm{C} ; \quad R_{f} 0.62 \quad(40 \%$ AcOEt/ petroleum ether); IR (KBr): 1233, 1461, 1552, 1617, $2198,3132 \mathrm{~cm}^{-1}{ }^{1} \mathrm{H}$ NMR $\left(500 \mathrm{MHz}, \mathrm{DMSO}-d_{6}\right): \delta$ $4.23\left(s, 3 \mathrm{H},-\mathrm{OCH}_{3}\right), 7.24-7.26(m, 2 \mathrm{H},-\mathrm{Ar}-H)$, 7.53-7.55 ( $m, 1 \mathrm{H},-\mathrm{Ar}-H), 7.63-7.64(m, 2 \mathrm{H},-\mathrm{Ar}-$ $H), 8.44-8.46(m, 1 \mathrm{H},-\mathrm{Ar}-H), 8.57(s, 1 \mathrm{H},-\mathrm{Ar}-H)$, 8.81-8.83 ( $m, 2 \mathrm{H},-\mathrm{Ar}-H), 12.18($ brs $, 1 \mathrm{H},-\mathrm{N} H)$; ${ }^{13} \mathrm{C}$ NMR (125 MHz, DMSO- $\left.d_{6}\right): \delta 56 \cdot 4,91 \cdot 5,96 \cdot 5$, $112 \cdot 6,113 \cdot 1,114 \cdot 3,117 \cdot 9,122 \cdot 5,122 \cdot 6,123 \cdot 8$, $123 \cdot 7,126 \cdot 3,132 \cdot 2,137 \cdot 1,142 \cdot 3,150 \cdot 7,158 \cdot 7$, 159.5, 164.7; MS (EI): $m / z 352.20\left[\mathrm{M}^{+}+\mathrm{H}^{+}\right]$; Anal. Calcd. for $\mathrm{C}_{21} \mathrm{H}_{13} \mathrm{~N}_{5} \mathrm{O}$ : C $71.79 \mathrm{H} 3.73 \mathrm{~N} 19.93$. Found: C 71.70 H 3.74 N 19.96.

$2.20 \quad 2-(1 H-I n d o l-3-y l)-6-m e t h o x y-4-(1-m e t h y l-1 H-$ indol-2-yl)pyridine-3,5-dicarbonitrile (table 1, entry 15) 40: Yellow solid; m.p. $242-245^{\circ} \mathrm{C} ; R_{f} 0.75$ (40\% AcOEt/Petroleum ether); IR (KBr): 1155 , $1227,1373,1437,1523,1566,2227,3285 \mathrm{~cm}^{-1}$ ${ }^{1} \mathrm{H}$ NMR $\quad\left(500 \mathrm{MHz}\right.$, DMSO- $\left.d_{6}\right): \delta 3.74(s, 3 \mathrm{H}$, $\left.-\mathrm{NCH}_{3}\right), 4.25\left(s, 3 \mathrm{H},-\mathrm{OCH}_{3}\right), 6.77(s, 1 \mathrm{H},-\mathrm{Ar}-\mathrm{H})$, $6.91(s, 1 \mathrm{H},-\operatorname{Ar}-H), 7 \cdot 11-7 \cdot 15(m, 2 \mathrm{H},-\operatorname{Ar}-H)$, 7.26-7.28 (m, 2H, -Ar-H), 7.53-7.57 (m, 2H, -Ar$H), 8.48-8.49(m, 1 \mathrm{H},-\mathrm{Ar}-H), 8.63(s, 1 \mathrm{H},-\mathrm{Ar}-H)$, $12.20(\mathrm{brs}, 1 \mathrm{H},-\mathrm{NH}) ;{ }^{13} \mathrm{C}$ NMR $(125 \mathrm{MHz}$, DMSO$\left.d_{6}\right): \delta 31 \cdot 6,56 \cdot 4,93 \cdot 2,98 \cdot 2,111 \cdot 1,111 \cdot 2,113 \cdot 1$, $114 \cdot 6,118 \cdot 1,120 \cdot 8,121 \cdot 6,122 \cdot 4,123 \cdot 6,123 \cdot 7$, $127 \cdot 3,132 \cdot 3,132 \cdot 7,137 \cdot 1,138 \cdot 7,152 \cdot 0,161 \cdot 6$, 164.9, 166.2; MS (EI): $m / z$ 404.33 $\left[\mathrm{M}^{+}+\mathrm{H}^{+}\right]$; Anal. Calcd. for $\mathrm{C}_{25} \mathrm{H}_{17} \mathrm{~N}_{5} \mathrm{O}$ : C $74.43 \mathrm{H} 4.25 \mathrm{~N}$ 17.36. Found: C 74.34 H 4.26 N 17.39.

2.2p 2,4-Di-1H-indol-3-yl-6-methoxypyridine-3,5dicarbonitrile (table 1, entry 16) 4p: Yellow solid; m.p. $230-233^{\circ} \mathrm{C} ; R_{f} 0.41(40 \%$ AcOEt/Petroleum ether); IR (KBr): 1147, 1227, 1304, 1519, 1555, $1611,2222,3263,3364 \mathrm{~cm}^{-1}{ }^{1} \mathrm{H}$ NMR $(500 \mathrm{MHz}$, DMSO- $\left.d_{6}\right): \delta 4.22\left(s, 3 \mathrm{H},-\mathrm{OCH}_{3}\right), 7 \cdot 13(t, 1 \mathrm{H}$, $J=6.9 \mathrm{~Hz}-\mathrm{Ar}-H), 7.21-7.24(m, 3 \mathrm{H},-\mathrm{Ar}-H), 7.52-$ $7.54(m, 2 \mathrm{H},-\operatorname{Ar}-H), 7.57(d, 1 \mathrm{H}, J=7.6 \mathrm{~Hz},-\mathrm{Ar}-$ 
$H), 7.96(s, 1 \mathrm{H},-\mathrm{Ar}-H), 8.44-8.46(m, 1 \mathrm{H},-\mathrm{Ar}-H)$, $8.59(s, 1 \mathrm{H},-\mathrm{Ar}-H), 10.89($ brs $, 1 \mathrm{H},-\mathrm{NH}), 11.87$ $(b r s, 1 \mathrm{H},-\mathrm{N} H) ;{ }^{13} \mathrm{C}$ NMR $\left(125 \mathrm{MHz}, \mathrm{DMSO}-d_{6}\right): \delta$ $56 \cdot 1,91 \cdot 7,97 \cdot 7,109 \cdot 0,112 \cdot 8,112 \cdot 9,113 \cdot 0,115 \cdot 6$, $119 \cdot 2,120 \cdot 5,120 \cdot 7,122 \cdot 1,122 \cdot 6,122 \cdot 8,123 \cdot 4$, $125 \cdot 3,126 \cdot 6,129 \cdot 0,132 \cdot 0,136 \cdot 7,137 \cdot 1,155 \cdot 3$, 159.8, 162.6, 165.5; MS (EI): $m / z 390 \cdot 20\left[\mathrm{M}^{+}+\mathrm{H}^{+}\right]$. Anal. Calcd. for $\mathrm{C}_{24} \mathrm{H}_{15} \mathrm{~N}_{5} \mathrm{O}$ : C $74.02 \mathrm{H} 3.88 \mathrm{~N}$ 17.98. Found: C 74.11 H 3.87 N 17.95.

\subsection{Synthesis of 6,6'-bis-(1H-indol-3-yl)-4-4'-} diaryl-[2,2']bipyridinyl-5,5'-dicarbonitrile

A mixture of dimedone $(2 \mathrm{mmol})$, cinnamil $(1 \mathrm{mmol})$ and $5 \mathrm{~g}$ of ammonium acetate under neat condition was refluxed at $120^{\circ} \mathrm{C}$ for appropriate time mentioned as in table 2. After the completion of the reaction (as monitored by TLC), it was poured into water and then filtered washed with acetone and then dried. The obtained crude solid was purified further by recrystallisation with DMF and appropriate isolated yield is shown in table 2 .

2.3a 7,7,7',7'-Tetramethyl-4,4'-diphenyl-4,6, 7,8,4', 6', 7',8'-octahydro- $1 \mathrm{H}, 1 \mathrm{H}$-[2,2 ] biquinolinyl-5,5'-

dione (Table 2, entry 1) 7a: Yellow solid; m.p. 293-296 ${ }^{\circ} \mathrm{C} ; R_{f} 0.81$ (40\% AcOEt/Petroleum ether); IR (KBr): 1126, 1314, 1384, 1485, 1587, 2934, $3340 \mathrm{~cm}^{-1}$; ${ }^{1} \mathrm{H}$ NMR $\left(500 \mathrm{MHz}, \mathrm{DMSO}-d_{6}\right): \delta 0.86$ $\left(s, 6 \mathrm{H},-\mathrm{CH}_{3}\right), 0.94\left(s, 6 \mathrm{H},-\mathrm{CH}_{3}\right), 1.88(\mathrm{Abq}, 4 \mathrm{H}$, $J=8.2 \mathrm{~Hz},-\mathrm{CH}), 2.35\left(s, 4 \mathrm{H},-\mathrm{CH}_{2}\right), 4.42(d, 2 \mathrm{H}$, $J=5.3 \mathrm{~Hz}$, dihydropyridyl-CH), $5.26 \quad(d, 2 \mathrm{H}$, $J=5.3 \mathrm{~Hz}$, dihydropyridyl-C $=\mathrm{CH}), 7.05(\mathrm{~m}, 2 \mathrm{H}, \mathrm{Ar}-$ $H), 7 \cdot 13(d, 4 \mathrm{H}, J=7.6 \mathrm{~Hz}, \operatorname{Ar}-H), 7 \cdot 18(m, 4 \mathrm{H}, \mathrm{Ar}-$ $H), 8.15($ brs $, 2 \mathrm{H},-\mathrm{NH}) ;{ }^{13} \mathrm{C}$ NMR $(125 \mathrm{MHz}$, DMSO- $d_{6}$ ): $\delta 27 \cdot 6,29 \cdot 2,29 \cdot 6,32 \cdot 6,38 \cdot 2,41 \cdot 8,50 \cdot 8$, $107 \cdot 2,117 \cdot 1,119 \cdot 3,127 \cdot 6,128 \cdot 2,128 \cdot 5,128 \cdot 8$, $128.9,129 \cdot 2,129 \cdot 8,131 \cdot 6,133 \cdot 5,136 \cdot 2,141 \cdot 3$, $143 \cdot 2,144 \cdot 8,150 \cdot 2,158 \cdot 2,162 \cdot 2,183 \cdot 1,195 \cdot 2$; MS (ESI LCQ-MS): $m / z 505.47\left[\mathrm{M}^{+}+\mathrm{H}^{+}\right]$. Anal. Calcd. for $\mathrm{C}_{34} \mathrm{H}_{36} \mathrm{~N}_{2} \mathrm{O}_{2}$ : C 80.92 H 7.19 N 5.55. Found: C $80.81 \mathrm{H} 7.22 \mathrm{~N} 5.52$.

$2.3 \mathrm{~b} \quad$ 7,7,7',7'-Tetramethyl-4,4'-bis(4-methylphenyl)$4,6,7,8,4^{\prime}, 6^{\prime}, 77^{\prime}, 8^{\prime}$-octahydro-1H,1H-[2,2']biquinolinyl5,5'-dione (table 2, entry 2) 7b: Yellow solid; m.p. $185-187^{\circ} \mathrm{C} ; R_{f} 0.84$ (40\% AcOEt/petroleum ether); IR (KBr): 1057, 1128, 1386, 1490, 1588, 2935, $3320 \mathrm{~cm}^{-1}{ }^{1} \mathrm{H}$ NMR $\left(500 \mathrm{MHz}, \mathrm{DMSO}-d_{6}\right): \delta 0.91$ $\left(s, 6 \mathrm{H},-\mathrm{CH}_{3}\right), 0.97\left(s, 6 \mathrm{H},-\mathrm{CH}_{3}\right), 1.93(\mathrm{Abq}, 4 \mathrm{H}$, $J=8.6 \mathrm{~Hz},-\mathrm{CH}), 2.19\left(s, 6 \mathrm{H}, \mathrm{Ar}-\mathrm{CH}_{3}\right), 2.29(s, 4 \mathrm{H}$,
$\left.-\mathrm{CH}_{2}\right), 4.58(\mathrm{~d}, 2 \mathrm{H}, J=6 \cdot 1 \mathrm{~Hz}$, dihydropyridyl- $\mathrm{C} H)$, $6.63(d, 2 \mathrm{H}, J=4.5 \mathrm{~Hz}$, dihydropyridyl- $\mathrm{C}=\mathrm{C} H)$, $7.18(m, 4 \mathrm{H}, \mathrm{Ar}-H), 7.67(d, 4 \mathrm{H}, J=7.6 \mathrm{~Hz}, \mathrm{Ar}-H)$, 8.55 (brs, $2 \mathrm{H},-\mathrm{NH}) ;{ }^{13} \mathrm{C}$ NMR $\left(125 \mathrm{MHz}, \mathrm{CDCl}_{3}\right)$ : $\delta 21 \cdot 0,21 \cdot 5,27 \cdot 5,29 \cdot 2,29 \cdot 6,32 \cdot 6,38 \cdot 1,41 \cdot 7,50 \cdot 7$, $107 \cdot 3,117 \cdot 1,119 \cdot 4,127 \cdot 9,128 \cdot 5,128 \cdot 8,128 \cdot 9$, $129 \cdot 3,129 \cdot 7,131 \cdot 7,133 \cdot 7,136 \cdot 3,141 \cdot 5,143 \cdot 3$, 144.9, 150.2, 183.2, 195.3; MS (ESI LCQ-MS): $\mathrm{m} / \mathrm{z}$ $533.47\left[\mathrm{M}^{+}+\mathrm{H}^{+}\right]$. Anal. Calcd. for $\mathrm{C}_{36} \mathrm{H}_{40} \mathrm{~N}_{2} \mathrm{O}_{2}$ : C 81.17 H 7.57 N 5.26. Found: C 80.28 H 7.52 N $5 \cdot 23$.

2.3c 7,7,7',7'-Tetramethyl-4,4'-bis(4-methoxyphenyl)$4,6,7,8,4^{\prime}, 6^{\prime}, 7^{\prime}, 8^{\prime}$-octahydro- $1 \mathrm{H}, 1 \mathrm{H}-[2,2 \mathrm{~J}$ biquinolinyl5,5'-dione (table 2, entry 3) 7c: Yellow solid; m.p. 226-228 ${ }^{\circ} \mathrm{C} ; R_{f} 0.6$ (40\% AcOEt/petroleum ether); IR (KBr): 1176, 1257, 1445, 1562, 3014, $3129 \mathrm{~cm}^{-1}$ ${ }^{1} \mathrm{H}$ NMR $\left(500 \mathrm{MHz}\right.$, DMSO- $\left.d_{6}\right): \delta 1.02 \quad(s, 6 \mathrm{H}$, $\left.-\mathrm{CH}_{3}\right), 1.08\left(\mathrm{~s}, 6 \mathrm{H},-\mathrm{CH}_{3}\right), 2 \cdot 15(\mathrm{Abq}, 4 \mathrm{H}, J=8 \cdot 1$ $\mathrm{Hz},-\mathrm{CH}), 2.36\left(\mathrm{~s}, 4 \mathrm{H},-\mathrm{CH}_{2}\right), 3.75(s, 6 \mathrm{H}$, Ar$\left.\mathrm{OCH}_{3}\right), 4.78(d, 2 \mathrm{H}, J=5.6 \mathrm{~Hz}$, dihydropyridyl$\mathrm{CH}), 6.24(\mathrm{~m}, 2 \mathrm{H}$, dihydropyridyl-C $=\mathrm{CH}), 6.81(\mathrm{~m}$, $4 \mathrm{H}, \operatorname{Ar}-H), 6.88(m, 2 \mathrm{H}, \mathrm{Ar}-H), 7.50(m, 2 \mathrm{H}, \mathrm{Ar}-H)$ $8.55($ brs $, 2 \mathrm{H},-\mathrm{NH}) ;{ }^{13} \mathrm{C}$ NMR $\left(125 \mathrm{MHz}, \mathrm{CDCl}_{3}\right)$ : $\delta 27 \cdot 5,28 \cdot 3,29 \cdot 2,29 \cdot 7,32 \cdot 6,37 \cdot 6,41 \cdot 7,50 \cdot 7,55 \cdot 2$, $55.4,107.4,113.6,113.9,114.4,115.7,119.0$, $127 \cdot 2,129 \cdot 1,129 \cdot 5,130 \cdot 4,133 \cdot 7,138 \cdot 6,144 \cdot 6$, $150 \cdot 1,158 \cdot 4,161 \cdot 9,183 \cdot 1,195 \cdot 3$; MS (ESI LCQMS): $m / z \quad 565.20 \quad\left[\mathrm{M}^{+}+\mathrm{H}^{+}\right]$. Anal. Calcd. for $\mathrm{C}_{36} \mathrm{H}_{40} \mathrm{~N}_{2} \mathrm{O}_{4}$ : C $76.57 \mathrm{H} 7.14 \mathrm{~N}$ 4.96. Found: C 76.66 $\mathrm{H} 7 \cdot 17 \mathrm{~N} 4.98$.

2.3d 7,7,7',7'-Tetramethyl-4,4'-bis(4-chlorophenyl)$4,6,7,8,4^{\prime}, 6^{\prime}, 7^{\prime}, 8^{\prime}$-octahydro- $1 \mathrm{H}, 1 \mathrm{H}-[2,2]$ biquinolinyl-5,5'-dione (table 2, entry 4) 7d: Yellow solid; m.p. $286-289^{\circ} \mathrm{C} ; R_{f} 0.81 \quad(40 \%$ AcOEt/petroleum ether); IR (KBr): 1319, 1384, 1484, 1590, 2928, $3352 \mathrm{~cm}^{-1}{ }^{1} \mathrm{H}$ NMR $\left(500 \mathrm{MHz}, \mathrm{DMSO}-d_{6}\right): \delta 0.85$ $\left(s, 6 \mathrm{H},-\mathrm{CH}_{3}\right), 0.95\left(s, 6 \mathrm{H},-\mathrm{CH}_{3}\right), 1.89(\mathrm{Abq}, 4 \mathrm{H}$, $J=9.2 \mathrm{~Hz},-\mathrm{CH}), 2.35\left(s, 4 \mathrm{H},-\mathrm{CH}_{2}\right), 4.46(d, 2 \mathrm{H}$, $J=4.6 \mathrm{~Hz}$, dihydropyridyl- $\mathrm{CH}), 5.52 \quad(d, 2 \mathrm{H}$, $J=4.6 \mathrm{~Hz}$, dihydropyridyl-C=CH), $7.14 \quad(m, 4 \mathrm{H}$, $J=8.4 \mathrm{~Hz}, \operatorname{Ar}-H), 7.25(d, 4 \mathrm{H}, J=8.4 \mathrm{~Hz}, \operatorname{Ar}-H)$, $8.22(b r s, 2 \mathrm{H},-N H) ;{ }^{13} \mathrm{C}$ NMR $(125 \mathrm{MHz}$, DMSO$\left.d_{6}\right): \delta 27 \cdot 3,28 \cdot 1,29 \cdot 0,29 \cdot 5,32 \cdot 4,37 \cdot 4,41 \cdot 5,50 \cdot 8$, $55 \cdot 1,55 \cdot 3,107 \cdot 2,113 \cdot 4,113 \cdot 8,114 \cdot 2,115 \cdot 5,119 \cdot 1$, $127 \cdot 1,129 \cdot 2,129.4,130 \cdot 5,133 \cdot 5,138.5,144 \cdot 5$, $150 \cdot 2,158 \cdot 2,161 \cdot 7,183 \cdot 3,195 \cdot 4$; MS (ESI LCQMS): $m / z \quad 573.20 \quad\left[\mathbf{M}^{+}+\mathrm{H}^{+}\right]$. Anal. Calcd. for $\mathrm{C}_{34} \mathrm{H}_{34} \mathrm{Cl}_{2} \mathrm{~N}_{2} \mathrm{O}_{2}$ : C 71.20 H 5.98 N 4.88. Found: $\mathrm{C}$ 71.28 H 5.95 N 4.87. 
2.3e 7,7,7',7'-Tetramethyl-4,4'-bis(3,4-dimethoxyphenyl)-4, 6, 7,8, 4', 6', 7', ' 'octahydro- $1 \mathrm{H}, 1 \mathrm{H}-\left[2,2^{\prime}\right]$ biquinolinyl-5,5'-dione (table 2, entry 5) 7e: Yellow solid; m.p. $211-214^{\circ} \mathrm{C} ; \quad R_{f} 0.35 \quad(40 \%$ AcOEt/ petroleum ether); IR (KBr): 1345, 1212, 1496, 1575, 2945, $3345 \mathrm{~cm}^{-1}{ }^{1} \mathrm{H}$ NMR $\left(500 \mathrm{MHz}\right.$, DMSO- $\left.d_{6}\right): \delta$ $1.07\left(s, 6 \mathrm{H},-\mathrm{CH}_{3}\right), 1 \cdot 12\left(\mathrm{~s}, 6 \mathrm{H},-\mathrm{CH}_{3}\right), 2 \cdot 12(\mathrm{Abq}$, $4 \mathrm{H}, J=8.6 \mathrm{~Hz},-\mathrm{CH}), 2.31\left(s, 4 \mathrm{H},-\mathrm{CH}_{2}\right), 3.68(s$, $\left.6 \mathrm{H}, \mathrm{Ar}-\mathrm{OCH}_{3}\right), 3.73\left(s, 6 \mathrm{H}, \mathrm{Ar}-\mathrm{OCH}_{3}\right), 4.76(d, 2 \mathrm{H}$, $J=5.6 \mathrm{~Hz}$, dihydropyridyl-CH), $6.21(m, 2 \mathrm{H}$, dihydropyridyl-C $=\mathrm{C} H), 6.85(m, 2 \mathrm{H}, \operatorname{Ar}-H), 6.94(m$, $2 \mathrm{H}, \operatorname{Ar}-H), 7.56(m, 2 \mathrm{H}, \mathrm{Ar}-H) 8.59$ (brs, 2H, $-\mathrm{N} H) ;{ }^{13} \mathrm{C}$ NMR $\left(125 \mathrm{MHz}, \mathrm{CDCl}_{3}\right): \delta 27 \cdot 8,28 \cdot 6$, $29 \cdot 4,29 \cdot 9,32 \cdot 8,37 \cdot 7,42 \cdot 3,51 \cdot 5,55 \cdot 8,56 \cdot 1,56 \cdot 2$, $56 \cdot 8,108 \cdot 1,113 \cdot 8,114 \cdot 0,114 \cdot 6,115 \cdot 8,119 \cdot 1$, $127 \cdot 2,129 \cdot 3,129 \cdot 7,130 \cdot 7,133 \cdot 9,138 \cdot 9,144 \cdot 5$, $150 \cdot 4,159 \cdot 3,162 \cdot 4,184 \cdot 5,196 \cdot 5$; MS (ESI LCQMS): $m / z \quad 625.40 \quad\left[\mathbf{M}^{+}+\mathrm{H}^{+}\right]$. Anal. Calcd for $\mathrm{C}_{38} \mathrm{H}_{44} \mathrm{~N}_{2} \mathrm{O}_{6}$ : C $73.05 \mathrm{H} \mathrm{7.10} \mathrm{N} \mathrm{4.48.} \mathrm{Found:} \mathrm{C} \mathrm{73.18}$ $\mathrm{H} 7 \cdot 16 \mathrm{~N} 4.52$.

2.3f 7,7,7',7'-Tetramethyl-4,4'-bis(4-bromophenyl)$4,6,7,8,4^{\prime}, 6^{\prime}, 7^{\prime}, 8^{\prime}$-octahydro- $1 \mathrm{H}, 1 \mathrm{H}$-[2,2 ']biquinolinyl-5,5'-dione (table 2, entry 6) 7f: Yellow solid; m.p. $282-284^{\circ} \mathrm{C} ; R_{f} 0.79(40 \%$ AcOEt/petroleum ether); IR (KBr): 1189, 1259, 1426, 1559, 2975, $3189 \mathrm{~cm}^{-1}{ }^{1} \mathrm{H}$ NMR $\left(500 \mathrm{MHz}\right.$, DMSO- $\left.d_{6}\right): \delta 0.89$ $\left(s, 6 \mathrm{H},-\mathrm{CH}_{3}\right), 0.95\left(\mathrm{~s}, 6 \mathrm{H},-\mathrm{CH}_{3}\right), 2.08(\mathrm{Abq}, 4 \mathrm{H}$, $J=8.1 \mathrm{~Hz},-\mathrm{CH}), 2.32\left(s, 4 \mathrm{H},-\mathrm{CH}_{2}\right), 4.91(d, 2 \mathrm{H}$, $J=5.6 \mathrm{~Hz}$, dihydropyridyl-CH), $6.21(\mathrm{~m}, 2 \mathrm{H}$, dihydropyridyl- $\mathrm{C}=\mathrm{C} H), 7 \cdot 08-7 \cdot 12(m, 2 \mathrm{H}, \mathrm{Ar}-\mathrm{H}), 7 \cdot 18$ $7.22(\mathrm{~m}, 4 \mathrm{H}, \mathrm{Ar}-\mathrm{H}), 7.48$ (m, 2H, Ar-H) 8.47 (brs, $2 \mathrm{H},-\mathrm{N} H) ;{ }^{13} \mathrm{C}$ NMR $\left(125 \mathrm{MHz}, \mathrm{CDCl}_{3}\right): \delta 26.8$, $27 \cdot 2,29 \cdot 1,29 \cdot 7,32 \cdot 4,37 \cdot 3,41 \cdot 5,50 \cdot 3,107 \cdot 1,112 \cdot 7$, $113 \cdot 5,114 \cdot 1,115 \cdot 3,118 \cdot 3,126 \cdot 3,128 \cdot 6 \cdot 6,129 \cdot 9$, $130 \cdot 7,133 \cdot 3,138 \cdot 3,144 \cdot 1,149 \cdot 8,157 \cdot 2,160 \cdot 5$, $182.9,194.3$; MS (ESI LCQ-MS): $\mathrm{m} / \mathrm{z} 663.28$ $\left[\mathrm{M}^{+}+\mathrm{H}^{+}\right]$. Anal. Calcd. for $\mathrm{C}_{34} \mathrm{H}_{34} \mathrm{Br}_{2} \mathrm{~N}_{2} \mathrm{O}_{4}$ : C 61.64 H 5.17 N 4.23. Found: C 61.75 H 5.15 N 4.21.

\section{Biological evaluation of newly synthesized compounds}

\subsection{Anti inflammatory activity of indol-3-yl pyri- dine derivatives}

The anti-inflammatory activities of various synthesized compounds were evaluated against carrageenaninduced paw oedema in rats. The inflammation was readily produced in rats in the form of paw oedema with the help of irritants such as carrageenan. Carra- geenan-induced paw oedema is a useful model to assess the contribution of mediators involved in vascular changes associated with acute inflammation. Carrageenan is sulphated polysaccharides obtained from sea weed (rhodophyceae) and when injected causes the release of histamine 5-HT, bradykininins and prostaglandins. It produce inflammation and oedema.

3.1a Preparation of $1 \%$ carrageenan solution: Carrageenan $(1 \mathrm{~g})$ was dissolved in $0.9 \%$ saline and the maximum volume administered to the rat was kept as $0.1 \mathrm{ml}$. The effects of drugs on paw oedema induced by carrageenan are shown in table 3. Almost all the drugs showed a maximum antiinflammatory effect and reduction in swelling. On the basis of these results, it can be concluded that greater anti-inflammatory activity was achieved with all the compounds, especially $\mathbf{4 h}, \mathbf{4 i}$ exhibit almost similar action as that of the standard. The antiinflammatory activity of the synthesized 3substituted indole derivatives was compared with indomethacin. The experiment was maintained up to four hours.

\subsection{Analgesic activity of bis(indol-3yl dihydropy- dine) derivatives}

The analgesic activities of various synthesized compounds were evaluated against acetic acid-induced writhing test-induced in Albinomice. Glacial acetic acid (GAA)-induced writhing 1\% GAA solution was injected (ip) and following this typical abdominal writhing response was observed. The number of writhes over a period of $20 \mathrm{~min}$ following GAA injection was counted. The data for drug treated groups were compared with appropriate controls for statistical significance. The results were analysed by student's ' $t$ ' test. The ' $t$ ' value of at least 0.05 or less was considered as level of significance.

3.2a Materials and methods: Animals: Albinomice 25-30 g; Drugs/chemicals: $1 \%$ acetic acid; 2\% Tween 80: Aspirin Std Drug $(50 \mathrm{~m} / \mathrm{kg})$; Synthetic compounds: $7 \mathbf{a}-\mathbf{f}$.

3.2b Acetic acid-induced writhing test ${ }^{5}$ : The prescreened animals were divided into 5 groups of 6 animals each. Aspirin in doses of $50 \mathrm{mg} / \mathrm{kg}$, suspended in $2 \%$ Tween 80 was used as the standard drug and administered orally. Writhing was induced 
Table 3. Anti-inflammatory activity of indol-3-yl pyridine derivatives.

\begin{tabular}{|c|c|c|c|c|c|c|c|c|c|c|}
\hline \multirow[b]{2}{*}{ Group } & \multirow[b]{2}{*}{$\begin{array}{l}\text { Doses } \\
(\mathrm{mg} / \mathrm{kg})\end{array}$} & \multicolumn{4}{|c|}{ Paw volume (mean $\pm \mathrm{SEM})$} & \multicolumn{5}{|c|}{ Percentage of inhibition } \\
\hline & & $0 \mathrm{~min}$ & $60 \mathrm{~min}$ & $120 \mathrm{~min}$ & $180 \mathrm{~min}$ & $240 \mathrm{~min}$ & $\begin{array}{c}60 \mathrm{~min} \\
(\%)\end{array}$ & $\begin{array}{c}120 \mathrm{~min} \\
(\%)\end{array}$ & $\begin{array}{l}180 \min \\
(\%)\end{array}$ & $\begin{array}{c}240 \mathrm{~min} \\
(\%)\end{array}$ \\
\hline $\begin{array}{l}\text { (1) } \\
\text { (control) }\end{array}$ & $\begin{array}{l}2 \cdot 5 \% \\
\text { Tween } 80\end{array}$ & $\begin{array}{l}0.682 \pm \\
0.033\end{array}$ & $\begin{array}{l}0.672 \pm \\
0.028\end{array}$ & $\begin{array}{l}0.674 \pm \\
0.025\end{array}$ & $\begin{array}{l}0.670 \pm \\
0.028\end{array}$ & $\begin{array}{l}0.663 \pm \\
0 \cdot 012\end{array}$ & 00 & 00 & 00 & 00 \\
\hline $\begin{array}{l}\text { (2) } \\
\text { Indomethacin }\end{array}$ & $\begin{array}{l}\text { Indomethacin } \\
10 \mathrm{mg} / \mathrm{kg}\end{array}$ & $\begin{array}{l}0.678 \pm \\
0.012\end{array}$ & $\begin{array}{l}0.632 \pm \\
0.032\end{array}$ & $\begin{array}{l}0.540 \pm \\
0.022\end{array}$ & $\begin{array}{l}0.430 \pm \\
0.010\end{array}$ & $\begin{array}{l}0.360 \pm \\
0.022\end{array}$ & $5 \cdot 95$ & $19 \cdot 88$ & 35 & $45 \cdot 7$ \\
\hline $4 a(3)$ & $20 \mathrm{mg} / \mathrm{kg}$ & $\begin{array}{l}0.667 \pm \\
0.010\end{array}$ & $\begin{array}{l}0.637 \pm \\
0.022\end{array}$ & $\begin{array}{l}0.573 \pm \\
0.031\end{array}$ & $\begin{array}{l}0.501 \pm \\
0.021\end{array}$ & $\begin{array}{l}0.380 \pm \\
0.032\end{array}$ & $5 \cdot 2$ & $14 \cdot 9$ & 25 & $42 \cdot 6$ \\
\hline $4 b(4)$ & $20 \mathrm{mg} / \mathrm{kg}$ & $\begin{array}{l}0.643 \pm \\
0.033\end{array}$ & $\begin{array}{l}0.622 \pm \\
0.032\end{array}$ & $\begin{array}{l}0.551 \pm \\
0.021\end{array}$ & $\begin{array}{l}0.460 \pm \\
0.032\end{array}$ & $\begin{array}{l}0.390 \pm \\
0.021\end{array}$ & $7 \cdot 4$ & $18 \cdot 2$ & $31 \cdot 3$ & $41 \cdot 7$ \\
\hline $4 c(5)$ & $20 \mathrm{mg} / \mathrm{kg}$ & $\begin{array}{l}0.651 \pm \\
0.022\end{array}$ & $\begin{array}{l}0.625 \pm \\
0.018\end{array}$ & $\begin{array}{l}0.543 \pm \\
0.018\end{array}$ & $\begin{array}{l}0.455 \pm \\
0 \cdot 018\end{array}$ & $\begin{array}{l}0 \cdot 385 \pm \\
0 \cdot 018\end{array}$ & $6 \cdot 9$ & $19 \cdot 4$ & 32 & $41 \cdot 9$ \\
\hline $4 d(6)$ & $20 \mathrm{mg} / \mathrm{kg}$ & $\begin{array}{l}0.668 \pm \\
0.012\end{array}$ & $\begin{array}{l}0.631 \pm \\
0.021\end{array}$ & $\begin{array}{l}0.550 \pm \\
0.032\end{array}$ & $\begin{array}{l}0.453 \pm \\
0.019\end{array}$ & $\begin{array}{l}0.400 \pm \\
0.012\end{array}$ & $6 \cdot 1$ & $18 \cdot 3$ & $32 \cdot 2$ & $39 \cdot 7$ \\
\hline $4 e(7)$ & $20 \mathrm{mg} / \mathrm{kg}$ & $\begin{array}{l}0.661 \pm \\
0.010\end{array}$ & $\begin{array}{l}0.621 \pm \\
0.018\end{array}$ & $\begin{array}{l}0.543 \pm \\
0.012\end{array}$ & $\begin{array}{l}0.451 \pm \\
0.031\end{array}$ & $\begin{array}{l}0.390 \pm \\
0.018\end{array}$ & $7 \cdot 5$ & $19 \cdot 4$ & $32 \cdot 6$ & $41 \cdot 2$ \\
\hline $4 f(8)$ & $20 \mathrm{mg} / \mathrm{kg}$ & $\begin{array}{l}0.643 \pm \\
0.033\end{array}$ & $\begin{array}{l}0 \cdot 616 \pm \\
0 \cdot 042\end{array}$ & $\begin{array}{l}0.533 \pm \\
0.018\end{array}$ & $\begin{array}{l}0.438 \pm \\
0.021\end{array}$ & $\begin{array}{l}0.380 \pm \\
0 \cdot 012\end{array}$ & $8 \cdot 3$ & $20 \cdot 9$ & $34 \cdot 6$ & $41 \cdot 2$ \\
\hline $4 g(9)$ & $20 \mathrm{mg} / \mathrm{kg}$ & $\begin{array}{l}0.657 \pm \\
0.022\end{array}$ & $\begin{array}{l}0.621 \pm \\
0.031\end{array}$ & $\begin{array}{l}0.551 \pm \\
0.019\end{array}$ & $\begin{array}{l}0.459 \pm \\
0.019\end{array}$ & $\begin{array}{l}0.390 \pm \\
0.031\end{array}$ & $7 \cdot 6$ & $18 \cdot 2$ & $31 \cdot 5$ & $41 \cdot 2$ \\
\hline $4 h(10)$ & $20 \mathrm{mg} / \mathrm{kg}$ & $\begin{array}{l}0.648 \pm \\
0.033\end{array}$ & $\begin{array}{l}0.612 \pm \\
0.021\end{array}$ & $\begin{array}{l}0.512 \pm \\
0 \cdot 018\end{array}$ & $\begin{array}{l}0.428 \pm \\
0 \cdot 042\end{array}$ & $\begin{array}{l}0.350 \pm \\
0.026\end{array}$ & $8 \cdot 9$ & 24 & $36 \cdot 1$ & $47 \cdot 2$ \\
\hline $4 \mathrm{i}(11)$ & $20 \mathrm{mg} / \mathrm{kg}$ & $\begin{array}{l}0.659 \pm \\
0.041\end{array}$ & $\begin{array}{l}0.621 \pm \\
0.026\end{array}$ & $\begin{array}{l}0.532 \pm \\
0.019\end{array}$ & $\begin{array}{l}0.430 \pm \\
0.032\end{array}$ & $\begin{array}{l}0.345 \pm \\
0.026\end{array}$ & $7 \cdot 6$ & 21 & 35.8 & $48 \cdot 0$ \\
\hline $4 \mathrm{j}(12)$ & $20 \mathrm{mg} / \mathrm{kg}$ & $\begin{array}{l}0.669 \pm \\
0.029\end{array}$ & $\begin{array}{l}0.627 \pm \\
0.038\end{array}$ & $\begin{array}{l}0.543 \pm \\
0.018\end{array}$ & $\begin{array}{l}0.452 \pm \\
0 \cdot 042\end{array}$ & $\begin{array}{l}0.390 \pm \\
0.038\end{array}$ & $6 \cdot 7$ & $19 \cdot 4$ & $32 \cdot 6$ & $41 \cdot 2$ \\
\hline $4 k(13)$ & $20 \mathrm{mg} / \mathrm{kg}$ & $\begin{array}{l}0.642 \pm \\
0.021\end{array}$ & $\begin{array}{l}0.613 \pm \\
0 \cdot 018\end{array}$ & $\begin{array}{l}0.531 \pm \\
0.032\end{array}$ & $\begin{array}{l}0.451 \pm \\
0.019\end{array}$ & $\begin{array}{l}0.385 \pm \\
0.032\end{array}$ & $8 \cdot 7$ & $21 \cdot 2$ & $32 \cdot 7$ & 42 \\
\hline $41(14)$ & $20 \mathrm{mg} / \mathrm{kg}$ & $\begin{array}{l}0.671 \pm \\
0.021\end{array}$ & $\begin{array}{l}0.620 \pm \\
0.011\end{array}$ & $\begin{array}{l}0.521 \pm \\
0.016\end{array}$ & $\begin{array}{l}0.450 \pm \\
0.033\end{array}$ & $\begin{array}{l}0.370 \pm \\
0.036\end{array}$ & $7 \cdot 7$ & $22 \cdot 7$ & $32 \cdot 8$ & $45 \cdot 0$ \\
\hline $4 m(15)$ & $20 \mathrm{mg} / \mathrm{kg}$ & $\begin{array}{l}0.670 \pm \\
0.021\end{array}$ & $\begin{array}{l}0.621 \pm \\
0.020\end{array}$ & $\begin{array}{l}0.520 \pm \\
0.020\end{array}$ & $\begin{array}{l}0 \cdot 448 \pm \\
0 \cdot 040\end{array}$ & $\begin{array}{l}0.385 \pm \\
0.012\end{array}$ & $7 \cdot 5$ & $22 \cdot 8$ & $33 \cdot 1$ & $42 \cdot 6$ \\
\hline $4 n(16)$ & $20 \mathrm{mg} / \mathrm{kg}$ & $\begin{array}{l}0.669 \pm \\
0.032\end{array}$ & $\begin{array}{l}0 \cdot 618 \pm \\
0 \cdot 022\end{array}$ & $\begin{array}{l}0.522 \pm \\
0.029\end{array}$ & $\begin{array}{l}0.440 \pm \\
0.022\end{array}$ & $\begin{array}{l}0.390 \pm \\
0.038\end{array}$ & $8 \cdot 0$ & $22 \cdot 5$ & $34 \cdot 3$ & $41 \cdot 2$ \\
\hline $40(17)$ & $20 \mathrm{mg} / \mathrm{kg}$ & $\begin{array}{l}0.649 \pm \\
0.020\end{array}$ & $\begin{array}{l}0.617 \pm \\
0.032\end{array}$ & $\begin{array}{l}0.513 \pm \\
0.033\end{array}$ & $\begin{array}{l}0.450 \pm \\
0.026\end{array}$ & $\begin{array}{l}0.380 \pm \\
0 \cdot 042\end{array}$ & $8 \cdot 2$ & $23 \cdot 8$ & $32 \cdot 8$ & $40 \cdot 4$ \\
\hline $4 p(18)$ & $20 \mathrm{mg} / \mathrm{kg}$ & $\begin{array}{l}0.662 \pm \\
0.019\end{array}$ & $\begin{array}{l}0.619 \pm \\
0.036\end{array}$ & $\begin{array}{l}0.532 \pm \\
0.030\end{array}$ & $\begin{array}{l}0.430 \pm \\
0.042\end{array}$ & $\begin{array}{l}0.365 \pm \\
0.032\end{array}$ & $7 \cdot 8$ & $21 \cdot 1$ & $35 \cdot 8$ & $44 \cdot 5$ \\
\hline
\end{tabular}

$10 \mathrm{~min}$ later by intraperetoneal injection of $0.1 \mathrm{ml}$ of $1 \%$ acetic acid in distilled water. The number of writhes was counted for $10 \mathrm{~min}$ immediately after the acetic acid injection. The percentage protection was calculated. Then the grouped animals received the standard and test drug as follows.
Group-1: $\quad$ Received subcutaneously 2\% Tween 80 and served as control.

Group-2: The standard group received intraperetoneally $50 \mathrm{mg} / \mathrm{kg}$ of the bodyweight of aspirin. 
Table 4. Analgesic activity of bis(indol-3yl dihydropydine) derivatives.

\begin{tabular}{|c|c|c|c|c|}
\hline Group & Animal weight (g) & Dose (mg/kg) & Writhing response & Inhibition (\%) \\
\hline 1 (control) & 25 & $1 \%$ Acetic acid & $38.00 \pm 0.70$ & 00 \\
\hline 2 (aspirin) & 30 & $50 \mathrm{mg} / \mathrm{kg}$ & $10.50 \pm 0.64$ & $72 \cdot 36$ \\
\hline $3(7 \mathbf{a})$ & 30 & $100 \mathrm{mg} / \mathrm{kg}$ & $12 \cdot 50 \pm 0.86$ & $67 \cdot 10$ \\
\hline $4(7 b)$ & 30 & $100 \mathrm{mg} / \mathrm{kg}$ & $11.75 \pm 0.75$ & $69 \cdot 07$ \\
\hline $5(7 c)$ & 30 & $100 \mathrm{mg} / \mathrm{kg}$ & $11.25 \pm 0.28$ & $70 \cdot 39$ \\
\hline $6(7 d)$ & 30 & $100 \mathrm{mg} / \mathrm{kg}$ & $12 \cdot 15 \pm 0.75$ & $68 \cdot 02$ \\
\hline $7(7 e)$ & 30 & $100 \mathrm{mg} / \mathrm{kg}$ & $11.25 \pm 0.75$ & $70 \cdot 22$ \\
\hline $8(7 f)$ & 30 & $100 \mathrm{mg} / \mathrm{kg}$ & $10.75 \pm 0.70$ & 71.71 \\
\hline
\end{tabular}
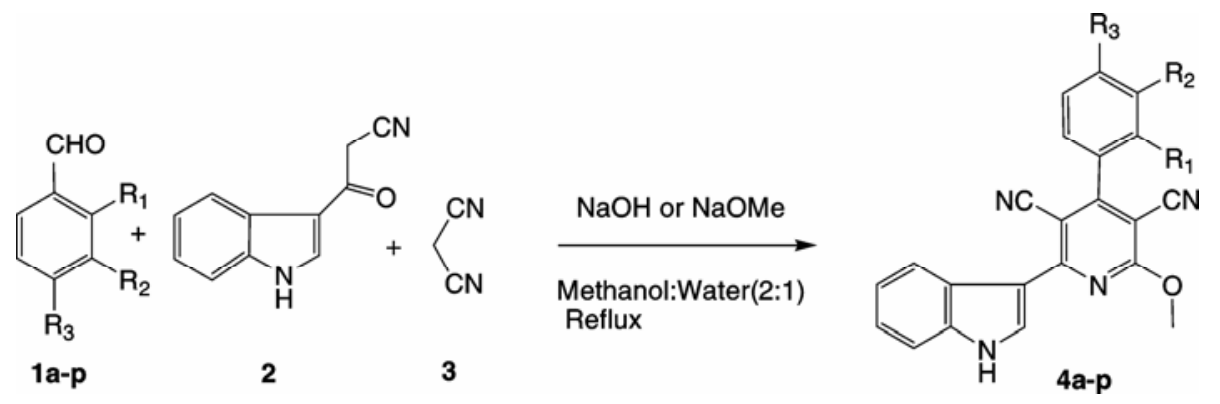

Scheme 1. Synthesis of indol-3-yl pyridine derivatives

Group-3-8: The 3-8 groups received drugs $\mathbf{7 a}-\mathbf{f}$ $(100 \mathrm{mg} / \mathrm{kg})$ respectively. All the above test compounds were dissolved in 2\% Tween 80 and administered orally. Writhing was induced $30 \mathrm{~min}$ later by intraperetoneal injections of $0.1 \mathrm{ml}$ of $1 \%$ acetic acid in distilled water the number of writhes was counted for 10 min immediately after the acetic acid injection. The percentage protection was calculated.

Writhing method is one of the most common tests for evaluating the analgesic efficacy of drugs/ compound in rodents. Narcotic analgesic inhibits both the types of pain, while NSAIDS such as paracetamol aspirin inhibit only the peripheral pain.

The abdominal constriction response induced by glacial acetic acid is a sensitive procedure to establish peripherally acting analgesics. This response is thought to involve local peritoneal receptors. The number of writhing observed during a $10 \mathrm{~min}$ period in control group was $38.00 \pm 0.70$ which corresponds with the finding of other workers.

The compounds $\mathbf{7 c}, \mathbf{7 e}$ and $\mathbf{7 f}$ significantly reduced the number of writhes. Analgesic activity of the synthesized bis-Hantzsch dihydropyridine derivatives was compared with aspirin and found to be having significant activity.

\section{Results and discussion}

In our previous report, we demonstrated that a mixture of substituted aldehyde, 3-cyanoacetyl indole and malononitrile in $\mathrm{InCl}_{3} /$ methanol under reflux condition for 2-6 h, afforded indol-3-yl pyridine derivatives in good yields. ${ }^{26}$ To further optimize conditions we extended the generality of our methodology, we explored an alternative procedure, which resulted in an operationally efficient process. Thus, instead of heating the reaction in $\mathrm{InCl}_{3} /$ methanol, the reaction mixture was refluxed in freshly prepared $\mathrm{NaOMe}$ (or) $\mathrm{NaOH}$ in methanol: water $(2: 1)$ ratio to give best yield of indol-3-yl pyridine derivatives in shorter reaction time. The isolation of the product was very simple and easy, without employing any purification technique like column chromatography. It was pretty much straightforward as the solid precipitated was filtered and then dried. The crude was further purified by recrystallisation with ethanol to afford highly pure substituted indol-3-yl pyridine derivative (scheme 1).

The substrate scope of the reaction under the optimized conditions was investigated, and the reaction was amenable to a wide variety of conditions on various aldehydes. On sequential addition of 
(a) Base catalysed mechanism

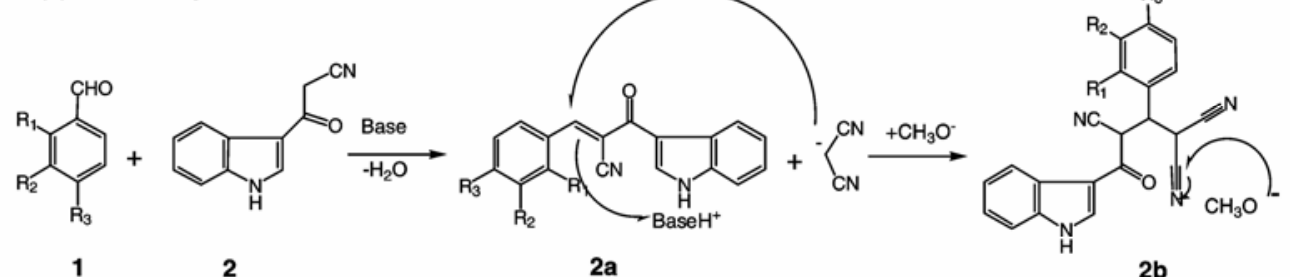

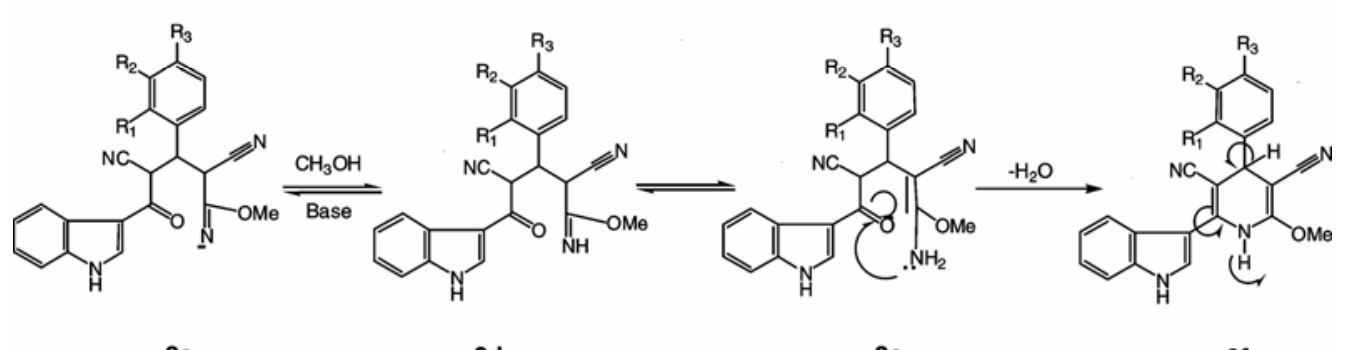

2c

2d

$2 e$

$2 f$

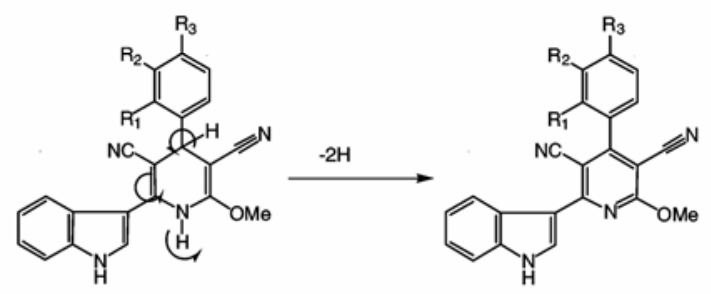

$2 f$

4

(b) Acid catalysed mechanism

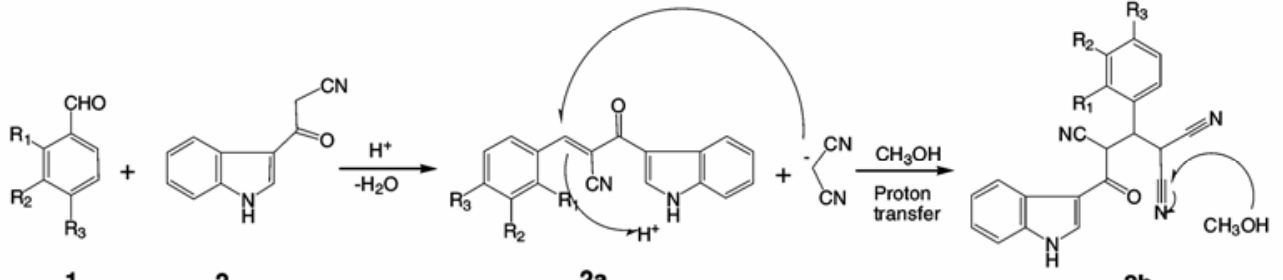

12

2a

2b

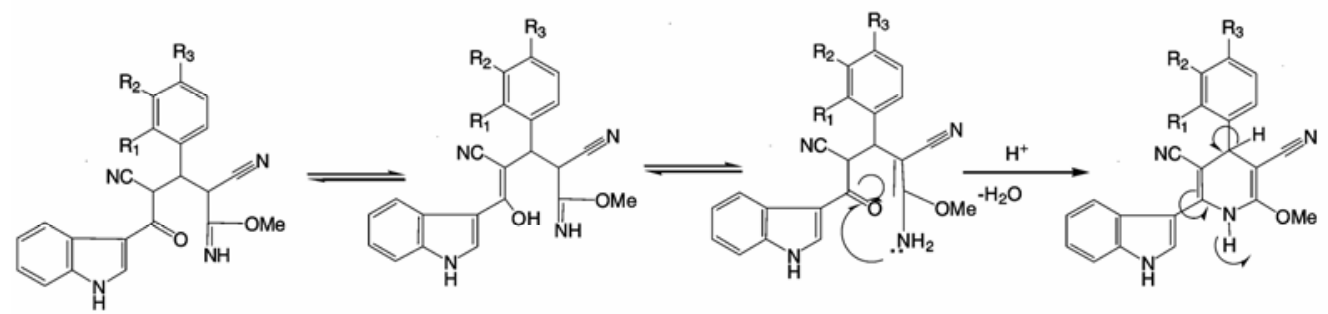

2c

2d

$2 e$

$2 f$

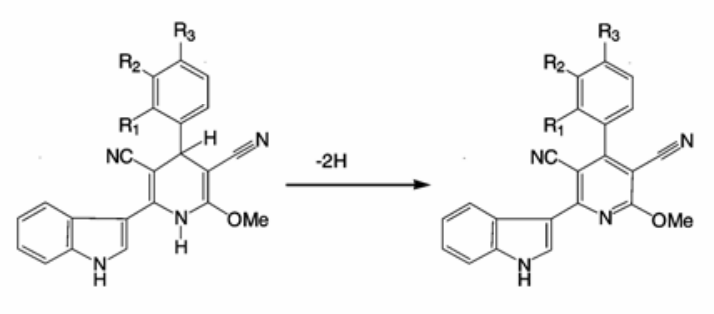

$2 f$

4

Scheme 2. A plausible mechanism for the formation of synthesis of indol-3-yl pyridine derivatives 

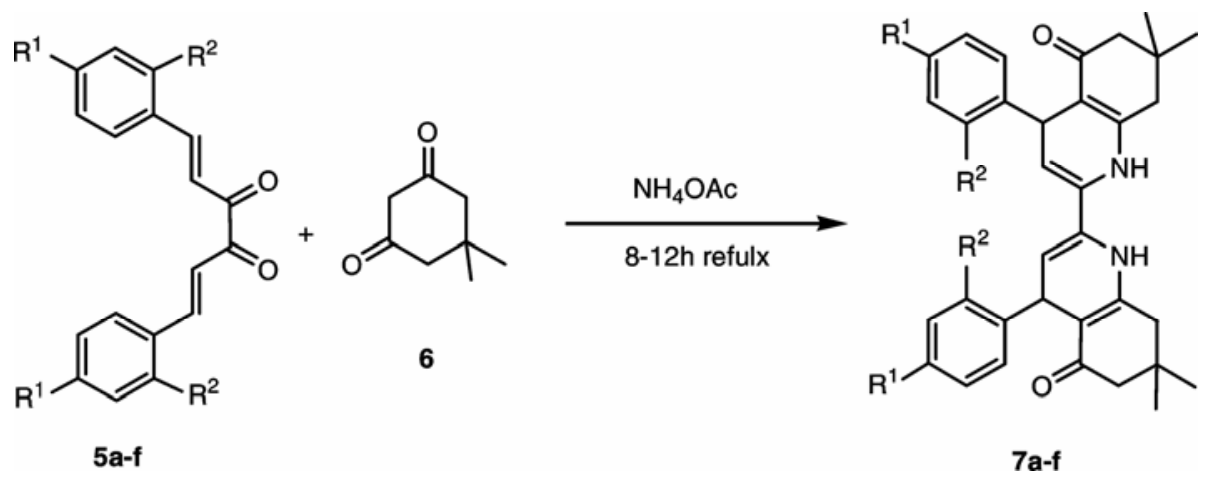

Scheme 3. Synthesis of $7,7^{\prime}, 7^{\prime}$-tetramethyl-4, $4^{\prime}$-bis(aryl)-4,6,7,8, $4^{\prime}, 6^{\prime}, 7^{\prime}, 8^{\prime}$ octahydro- $1 \mathrm{H}, 1 \mathrm{H}-\left[2,2^{\prime}\right]$ biquinolinyl-5,5'-dione derivatives.

the substrates under optimized conditions, the reaction proceeded smoothly with a wide range of functionalized aldehydes, including those containing ether nitro, halogens and polyaromatic spacers and the results are summarized in table 1 .

Based on the above results, a plausible base and acid catalysed mechanism was proposed for the formation indol-3-yl pyridine derivatives (scheme 2). ${ }^{7}$ Initially, 3-cyano acetyl indole reacts with corresponding aldehyde (1a-k) to give a $\alpha, \beta$ unsaturated ketone (2a) and on further it reacts with malononitrile (2b) followed by methoxide or methanol attack (2c) to give an intermediate (2d and 2e), which upon protonation yields Hantzsch dihydro pyridine derivative (2g). The later deprotonation afford to yield pyridine (4) derivative. However in the reverse reaction, malononitrile is first allowed to react with the corresponding aldehyde followed by 3cyanoacetyl indole attack did not lead to the formation of corresponding pyridine derivatives (scheme 2).

The structures of the compounds $\mathbf{4 a - p}$ were established through IR, ${ }^{1} \mathrm{H}$ NMR, ${ }^{13} \mathrm{C}$ NMR and elemental analysis as exemplified for compound $\mathbf{4 g}$ as follows: In the IR spectrum absorptions at 3222 and $2253 \mathrm{~cm}^{-1}$ supported the presence of $-\mathrm{NH}$ and $-\mathrm{C} \equiv \mathrm{N}$ functional groups. The ${ }^{1} \mathrm{H}$ NMR spectrum exhibited a broad distinguishable singlet at $\delta 12.14 \quad\left(\mathrm{D}_{2} \mathrm{O}\right.$ exchangeable) for $-\mathrm{NH}$ protons and a sharp distinguishable singlet appeared at $\delta 4.22$ for methoxy protons. Aromatic protons were seen in the range of $\delta$ 7.22-8.56. A distinctive peak at $\delta 55.7$ in the

${ }^{13} \mathrm{C}$ NMR spectrum confirmed the presence of methoxy group. The two cyano groups attached carbon characteristic peaks were appeared at $\delta 91.3$ and 96.6. The mass spectrum displayed the molecular ion $[\mathrm{M}+\mathrm{H}]^{+}$peak at $m / z 431 \cdot 3$. The structure of the compound $\mathbf{4 g}$ was further established by single crystal X-ray diffraction analysis (figure 2$)^{27}$.

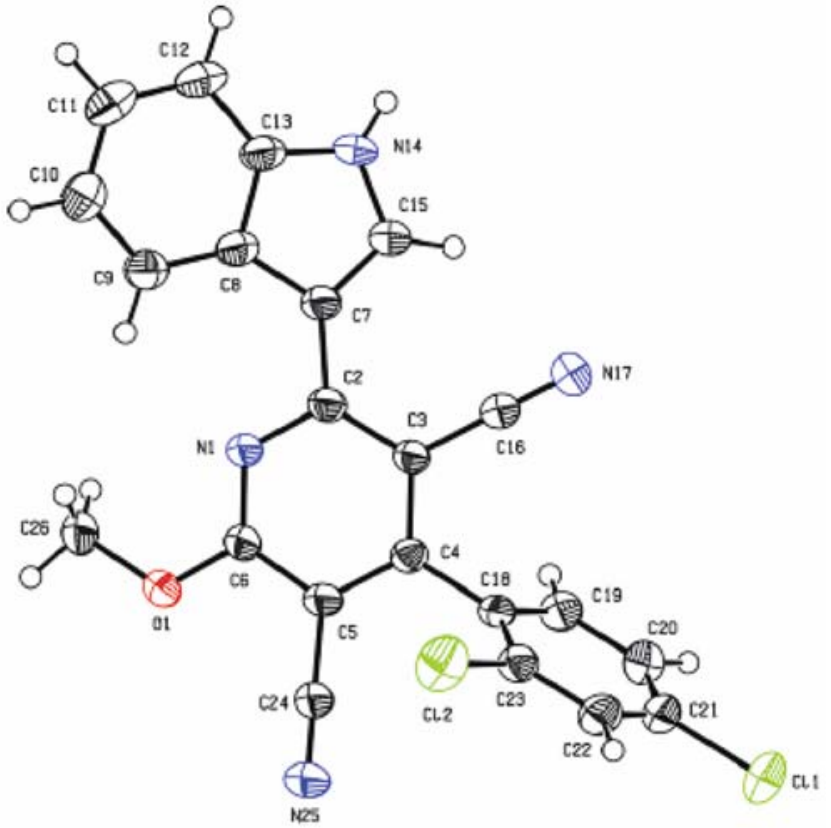

Figure 2. ORTEP diagram of compound $\mathbf{4 g}$.

Based on above results, we extended our protocol to the reaction with 1,6-diarylhexa-1,5-diene3,4dione, dimedone and ammonium acetate under reflux condition which gave bis(indol-3yl dihydropydine) derivatives in moderate yields (scheme 3 ). Under optimized conditions, the reaction proceeded smoothly with a wide range of functionalized cinnamils, including those containing ethers, chlorine and hydrocarbons and the results are summarized in table 2 .

The structure of compounds (7a-f) were examined based on the detailed spectroscopic studies as exemplified for compound $\mathbf{7 b}$ as follows: In the IR spectrum of compound (7b) showed stretching frequencies at 3320 and $1588 \mathrm{~cm}^{-1}$ for $-\mathrm{NH}$ and $\mathrm{C}=\mathrm{O}$ bonds. The ${ }^{1} \mathrm{H}$ NMR spectrum showed chemical 
shift of $\delta: 8.55$ (brs, $\mathrm{D}_{2} \mathrm{O}$ exchangeable) corresponds to $\mathrm{NH}$ protons. The doublet in chemical shift region of $\delta: 4.78$ and 6.63 corresponds to dihydropyridine ring protons. The sharp two singlets appeared in the region if $\delta: 183.2$ and 195.3 correspond to carbonyl carbons. The aromatic carbons resonated in the region of $\delta$ : 117.1-150.2. The mass spectrum showed distinguishable singlet peak at $m / z$ 533.47.

\section{Conclusions}

In conclusion, we have synthesized indol-3-yl pyridine in better yields via one pot multi-component reaction. These compounds showed good antiinflammatory activity in comparison with the standard drug indomethacin. Also, we have prepared series of bis-Hantzsch dihydropyridine derivatives. These compounds showed a good analgesic activity when compared with asprin. Further studies to delineate the scope and limitations of the present methodology are underway.

\section{References}

1. (a) Chebanov VA, Muravyova E A, Desenko S M, Musatov V I, Knyazeva I V, Shishkina S V, Shishkin O V and Kappe C O 2006 J. Comb. Chem. 8 427; (b) Dondoni A, Massi A, Sabbatini S and Bertolasi V J. Org. Chem. 2002676979

2. (a) Nilsson B L and Overman LE $2006 \mathrm{~J}$. Org. Chem. 71 7706; (b) Vugts D J, Koningstein M M, Schmitz R F, de Kanter F J, Groen M B and Orru R V 2006 Chem. Eur. J. 127178

3. Xu L W, Xia C G and Li L 2004 J. Org. Chem. 69 8482

4. Fewell S W, Smith C M, Lyon M A, Dumitrescu T P, Wipf P, Day B W and Brodsky J L $2004 \mathrm{~J}$. Biol. Chem. 27951131

5. (a) Chebanov V A, Saraev VE, Desenko S M, Chernenko V N, Shishkina S V, Shishkin O V, Kobzar K M and Kappe C O 2007 Org. Lett. 9 1691; (b) De Silva, R A, Santra S and Andreana P R 2008 Org. Lett. 104541

6. (a) Dondoni A, Massi A, Sabbatini S, Bertolasi V 2002 J. Org. Chem. 67 6979; (b) Glasnov T N, Stadlbauer W and Kappe C O 2005 J. Org. Chem. 703864
7. Evans C G and Gestwicki JE 2009 Org. Lett. 11 2957

8. Bringmann G, Reichert Y and Kane V V 2004 Tetrahedron 603539

9. Zhou Y, Kijima T, Kuwahara S, Watanabe M and Izumi T 2008 Tetrahedron Lett. 493757

10. Franco L H, Joffe E B K, Puricelly L, Tatian M, Seldes A M and Palermo J A 1998 J. Nat. Prod. 61 1130

11. (a) Farhanullah, Agarwal N, Goel A and Ram V J 2003 J. Org. Chem. 68 2983; (b) Joule J A, Smith G, Mills K 1995 Heterocyclic chemistry (London: Chapman and Hall) 3rd edn, pp. 72-11

12. (a)Teague S J 2008 J. Org. Chem. 73 9765; (b) Movassaghi M, Hill MD and Ahmad O K $2007 J$. Am. Chem. Soc. 12910096

13. Cooke M W and Hanan G S 2007 Chem. Soc. Rev. 36 1466

14. Constable E C 2007 Chem. Soc. Rev. 36246

15. (a) Kurth D G and Higuchi M 2006 Soft Matter 2 915; (b) Medlycott E A and Hanan G S 2005 Chem. Soc. Rev. 34 133; (c) Andres P R and Schubert U S 2004 Adv. Mater. 161043

16. Gribble G W 2000 J. Chem. Soc., Perkin Trans. I 1045

17. Xiong W N, Yang C G and Jiang B 2001 Bioorg. Med. Chem. 91773

18. Zhu S, Ji S, Su X, Sun C and Liu Y 2008 Tetrahedron Lett. 491777

19. Farghaly A M, Habib N S, Khalil M A, Sayed O A E and Alexandria 1989 J. Pharm. Sci. 390

20. Franco L H, Joffe E B K, Puricelly L, Tatian M, Seldes A M and Palermo J A 1998 J. Nat. Prod. 61 1130

21. Marchalin S and Kuthan J 1985 Czech. Chem. Commun. $\mathbf{5 0} 1862$

22. Radwana M A A and El-Sherbiny M 2007 Bioorg. Med. Chem. 151206

23. (a) Sun C, Jun Ji S and Liu Y 2007 Tetrahedron Lett. 48 8987; (b)Radwan M A A, Ragab E A, Sabrya N M and El-Shenawyc S M 2007 Bioorg. Med. Chem. 15 3832

24. Abdallah T A 2007 J. Heterocyclic Chem. 44961

25. Zaki ME A and Proenc MF 2007 Tetrahedron 63 3745

26. Thirumurugan P and Perumal P T 2009 Tetrahedron 657620

27. Thirumurugan P and Perumal P T 2009 Tetrahedron Lett. 504145

28. Thirumurugan $\mathrm{P}$, Muralidharan $\mathrm{D}$ and Perumal $\mathrm{P} T$ 2009 Dyes and Pigments $\mathbf{8 1} 245$ 